\title{
Classification of Non-normal Quartic Surfaces
}

\author{
Tohsuke URABE \\ Tokyo Metropolitan University
}

\section{Introduction}

Recently I am interested in quartic surfaces in $\boldsymbol{P}^{3}$, because I believe that there is a simple law explaining how singularities appear on quartic surfaces. Even though I have found out a law described by Dynkin graphs for a restricted class of quartic surfaces, it is very important to find out the general law. (Urabe [30], [31], Umezu-Urabe [29]). For this purpose it is necessary to study what kinds of singularities appear on non-normal quartic surfaces, as one step.

In this article we give the classification of non-normal quartic surfaces (i.e., surfaces of degree 4 with 1-dimensional singular loci) in the three dimensional projective space $\boldsymbol{P}^{3}$ over the complex number field $\boldsymbol{C}$.

Note that we can assume without loss of generality that the surface is reduced and irreducible because otherwise by considering its components we can reduce the problem to the case where degree is 1,2 or 3 . Classification of linear and quadratic surfaces is trivial and classification of cubic surfaces was given in Bruce-Wall [4].

We have many classical papers and modern ones treating this subject. (Clebsch [5], Jessop [12], Klein [16], [17], Kummer [18], Nöther [19], Rohn [21], Segre [23]; Kato-Naruki [14], [15], Shah [24], Takahashi-WatanabeHiguchi [26], Umezu [27], [28] etc.) For example, Jessop [12] was published in 1916, which treats quartic surfaces which are not ruled surfaces. We can find in it explanation of surfaces corresponding to the items (III-A-1), (III-B), and (III-C) in the next section. But it is not clear whether they knew that there are no other non-normal quartic surfaces than those in this article or not. Therefore in order to find out the law it is not enough. Moreover it is important to give a description from the modern view-point, because we can now use powerful tools developped during the 20 -th century such as sheaf theory, cohomology theory, etc. It seems 
that we can deduce something new owing to these tools. (For example, the results in section 5 and 6. .)

Here it should be mentioned that we have a very eminent theory of polarized varieties due to T. Fujita. (Fujita [8], [9], [10].) The notion of $\Delta$-genera is the main tool in his theory. The quartic surfaces treated in this article correspond to the case $0 \leqq \Delta \leqq 2$. Therefore it should be noted that most of items in our classification list can be found in Fujita's theory. But his results are not enough for our purpose, either, because of the following reason: Main objects of his classification are pairs $(M, L)$ where $M$ is a smooth manifold and $L$ is a line bundle over $M$. He assumed that $L$ is ample in his important parts. But if we try to treat singular surfaces, we cannot assume that it is ample. In this case $L$ is at most numerically effective. We sometimes have to prove in a more complicated way that some conclusions in his theory hold under this weaker condition. (See accounts from Lemma 4.2 to Proposition 4.5 in Section 4.) Indeed some items in our classification list cannot be found in his theory. (ruled surfaces over curves of genus 2.) However, of course we use 4 -genera as a powerful tool even in this article.

I would like to show in the forthcomming paper that possible combination of singularities on the surface belonging to (III-C) can be described in a simple method related to the Dynkin graph $D_{8}$. I have written this article as the preliminary part of the forthcomming one. I give a rough description of singularities on non-normal quartic surfaces.

Now we would like to give several remarks here. First Segre [23] has given the precise classification for surfaces belonging to the item (III-B) in the next section. Secondly there is a modern article which claims that any possible combination of isolated singularities on surfaces (III-B) corresponds to a proper subgraph of the extended Dynkin graph of type $D_{5}$ (Pinkham [20]). Thirdly, we would like to explain briefly the relation between this article and Swinnerton-Dyer's paper [25] "An enumeration of all varieties of degree 4". In Swinnerton-Dyer's one, the first item is "the hyper-surfaces of degree 4 in $P^{n}$ ". Thus the list in the next section gives one part of the precise description of the first item in the classification due to him.

In Section 1 we state the result of the classification. Section 2 in this article is the preliminary part. We explain that the minimal resolution $Z$ of an irreducible non-normal quartic surface $X$ is birationally equivalent to either (I) a ruled surface over a curve of genus 2, (II) a ruled surface over an elliptic curve or (III) a rational surface. Since we cannot apply Fujita's theory directly for the lack of ampleness, we 
devide the problem into these three cases. Moreover we show in Case (I) the surface $X$ is a cone over a singular plane quartic curve. In section 3 we discuss Case (II). Applying a proposition due to Fujita related to the Albanese mapping and its fibres, we give the complete classification in this case. The Case (III) of rational surfaces is treated in section 4. If $\Delta=0$ or 1 , we can apply Fujita's theory and we obtain the result in this case. The case of rational surfaces with $\Delta=2$ is dealt with separatedly. But studying the intersection of divisors, we complete the classification. In section 5 we treat a more delicate problem, that is, whether the rational surface can be blown-down to $\boldsymbol{P}^{2}$. Section 6 is the continuation of section 5 . We discuss blowing-downs to $\boldsymbol{P}^{2}$ with special properties.

We would like to express heartily thanks to my colleagues in Tokyo Metropolitan University for stimulating atmosphere.

Here we explain an important notion in the following sections. Let $E$ be a vector bundle of rank $r$ over a variety $V$. Each fibre $E_{x}^{\vee}$ of the dual bundle $E^{\vee}$ over a point $x \in V$ has an action of the multiplicative group $C^{*}=C-\{0\}$ defined by the multiplication. The quotient space of $E^{\vee}$ minus the zero-section by $C^{*}$ is denoted by $\boldsymbol{P}(E)$. This defines a fibre bundle $\pi: P(E) \rightarrow V$ whose fibre is a projective space of dimension $r-1$. The space $\boldsymbol{P}(E)$ carries a line bundle $L$ called the tautological line bundle and a surjective homomorphism $t: \pi^{*} E \rightarrow L$ of vector bundles. They have the following universal mapping property: For any open set $U \subset V$ and for any surjective homomorphism $\left.E\right|_{U} \rightarrow M$ to a line bundle $M$ over $U$, there are a unique section $s: U \rightarrow \boldsymbol{P}(E)$ (a morphism of varieties such that $\pi s$ is the identity) and an isomorphism $s^{*} L \cong M$ such that the composition $\left.E\right|_{U}=s^{*} \pi^{*} E \stackrel{s^{*}(t)}{\longrightarrow} s^{*} L \cong M$ coincides with the given homomorphism $\left.E\right|_{U} \rightarrow M$.

\section{$\S 1$. The results.}

Any non-normal reduced irreducible quartic surface $X$ in $\boldsymbol{P}^{3}$ is one of the following. We assume that every variety is defined over $C$. Let $H$ denote the intersection of $X$ and a general plane in $\boldsymbol{P}^{3}$.

(I) a cone over a singular plane quartic curve.

(II) a ruled surface over an elliptic curve constructed in the following way. (II-1) 〈necessary data〉 (1) a smooth irreducible elliptic curve $G$ (2) a non-trivial line bundle $N$ over $G$ with $\operatorname{deg} N=0$. (3) a line bundle $M$ over $G$ with $\operatorname{deg} M=2$.

〈method of construction〉 Let $\pi: Z=P\left(\mathcal{O}_{G} \oplus N\right) \rightarrow G$ be the projective bundle associated with the vector bundle $\mathcal{O}_{G} \oplus N$. Let $D_{1}$ and $D_{2}$ be the 
sections of $\pi$ associated with projections $\mathcal{O}_{a} \oplus N \rightarrow \mathcal{O}_{G}$ and $\mathcal{O}_{G} \oplus N \rightarrow N$ respectively. Set $L=\mathcal{O}_{Z}\left(D_{1}\right) \otimes \pi^{*} M$. The line bundle $L$ defines a morphism $\phi=\varphi_{L}: Z \rightarrow P^{3}$. The image $X=\varphi(Z)$ is the desired surface.

〈property〉 Set $L_{i}=\varphi\left(D_{i}\right)$ for $i=1,2$. The singular locus of $X$ coincides with $L_{1} \cup L_{2} . \quad L_{1}$ and $L_{2}$ are two lines in twisted position. The planesection $H$ has $A_{1}$-singularities at $H \cap L_{1}$ and $H \cap L_{2}$. The image by $\varphi$ of any fibre of $\pi$ is a line intersecting with $L_{1}$ and $L_{2}$.

(II-2) 〈necessary data〉 (1) an irreducible smooth elliptic curve $G$. (2) a line bundle $M$ over $G$ with $\operatorname{deg} M=2$.

〈method of construction〉 Let $E$ be a vector bundle of rank 2 which has a non-splitting exact sequence$$
0 \longrightarrow \mathcal{O}_{a} \longrightarrow E \longrightarrow \mathcal{O}_{a} \longrightarrow 0 \text {. }
$$

Let $\pi: Z=\boldsymbol{P}(E) \rightarrow G$ be the associated projective bundle. Note that $\pi$ has a section $s: G \rightarrow Z$ associated with the sequence $\left({ }^{*}\right)$. Let $D_{1}=s(G)$. Let $L=\mathcal{O}_{Z}\left(D_{1}\right) \otimes \pi^{*} M$. The line bundle $L$ defines a morphism $\varphi=\varphi_{L}: Z \rightarrow P^{s}$. The image $X=\varphi(Z)$ is the desired surface.

〈property〉 The singular locus of $X$ is the line $\phi\left(D_{1}\right)=L_{1}$. The planesection $H$ has a $A_{8}$-singular point at $H \cap L_{1}$. Any fibre of $\pi$ is sent by $\varphi$ to a line intersecting with $L_{1}$.

Furthermore for surfaces in (II-1), (resp. (II-2)), for every choice of $(G, M, N)$ (resp. $(G, M)$ ) satisfying the conditions in the above, there is a quartic surface corresponding to it.

(III) a rational surface $X \subset P^{3}$ which is described in one of the following items.

(III-A) $X$ is the image of a smooth surface $Z \subset P^{r}$ by a projection $\boldsymbol{P}^{\boldsymbol{s}} \cdots \rightarrow \boldsymbol{P}^{3}$ whose center is a line disjoint from $Z$. Here, $Z$ is one of the following. In this case the non-singular model $\widetilde{H}$ of the planesection $H$ is an irreducible rational curve. $X$ has no isolated singular points.

(A-1) $Z=\boldsymbol{P}^{2}$ and the embedding is the Veronese embedding associated with quadratic forms. (Steiner's Roman surface)

(A-2) $Z=P^{1} \times P^{1}$. The embedding $Z \rightarrow P^{5}$ is the one associated with the divisor $\bar{C}+2 \bar{F}$.

(A-3) $Z=\Sigma_{2}$ and the embedding associated with the divisor $\bar{C}+\bar{F}$.

Here for the Hirzebruch surface $\tau: \Sigma_{m}=\boldsymbol{P}\left(\mathcal{O}_{\mathbf{p}} \oplus \mathcal{O}_{\mathbf{p} 1}(m)\right) \rightarrow \boldsymbol{P}^{1} \quad(m=$ $0,1,2, \cdots)$, we denote by $\bar{C}$ the section of $\tau$ with $\bar{C}^{2}=m$ and by $\bar{F}$ the general fibre of $\tau$.

(III-B) $X$ is the image of the normal surface $\hat{X} \subset P^{4}$ with at most rational double points as singularities by a projection $\boldsymbol{P}^{4} \ldots \rightarrow \boldsymbol{P}^{\mathbf{s}}$ whose center is a point outside $\hat{X}$. Here $\hat{X}$ is a degenerate Del Pezzo surface of degree 
4, which is obtained as follows. Let $Z$ be a surface obtained from $\boldsymbol{P}^{2}$ by a successive 5 blowing-ups whose centers are in almost general position. (Demazure [6]). Let $\boldsymbol{Z} \rightarrow \boldsymbol{P}^{4}$ be the morphism associated with the anticanonical divisor $-K_{z}$. The image is $\hat{X}$. The strict transform $\widetilde{H}$ of $H$ on $Z$ is smooth and it is an irreducible elliptic curve.

(III-C) $X$ is a rational surface whose embedding is defined by a complete linear system on its normalization $\hat{X}$. In this case the one-dimensional singular locus is a line. $X$ may have isolated singular points outside the one-dimensional singular locus. Let $Z$ denote the minimal resolution of singularities of the normalization $\hat{X}$. The strict transform $\widetilde{H}$ on $Z$ of $H$ is smooth and $\widetilde{H}$ is an irreducible curve of genus 2 . A characteristic property of $Z$ is that the anti-canonical linear system $\left|-K_{Z}\right|$ of $Z$ contains only one element $D \in\left|-K_{Z}\right|$. The surface $Z$ is obtained from $P^{2}$ by successive 9 blowing-ups. The morphism $Z \rightarrow \boldsymbol{P}^{3}$ is defined by the divisor $\widetilde{H} \sim$ $4 \widetilde{E}_{0}-2 \bar{E}_{1}-\widetilde{E}_{2}-\widetilde{E}_{3}-\widetilde{E}_{4}-\widetilde{E}_{5}-\widetilde{E}_{8}-\widetilde{E}_{7}-\widetilde{E}_{8}-\widetilde{E}_{9}$, where $\widetilde{E}_{0}$ is the total transform of a line by the composition of blowing-ups $Z \rightarrow \boldsymbol{P}^{2}$ and $\widetilde{E}_{i}$ is the total transform of the exceptional curve of the $i$-th blowing-up for $i>0$. (The symbol $\sim$ denotes the linear equivalence.)

〈property〉 Any singular point on $\hat{X}$ which is not a rational double point lies on the inverse image of the 1-dimensional singular locus of $X$ and it is a rational triple point. The number of rational triple points is at most 2. The images on $X$ of these triple points on $\hat{X}$ are also triple points.

In the above, the curve singularity whose local defining equation is $x^{2}-y^{k+1}=0$ is called $A_{k}$-singularity. As for ratioal double points, please see Durfee [7] or Artin [2].

\section{§2. Preliminaries.}

Let $X \subset \boldsymbol{P}^{3}$ be a reduced irreducible non-normal quartic surface over $C$. Let $\nu: \hat{X} \rightarrow X$ be the normalization and $\rho: Z \rightarrow \hat{X}$ be the minimal resolution of singularities on $\hat{X}$. Note that the strict transform $\widetilde{H} \subset Z$ of the general plane section $H \subset X$ by $\nu \rho$ is smooth by the Bertini theorem.

Note moreover that every local ring $\mathcal{O}_{X, x}, x \in \hat{X}$ of $\hat{X}$ is Cohen-Macaulay since it is normal with dimension $\leqq 2$. We can define dualizing sheaves, $\omega_{X}, \omega_{\hat{X}}$, and $\omega_{z}$. (Altman-Kleiman [1].) The sheaf $\omega_{X}$ is trivial since $X$ is quartic.

Lemma 2.1. There is a non-zero effective divisor $\widetilde{D}$ on $Z$ with the following properties.
(a) $\omega_{z} \cong \mathscr{O}_{z}(-\widetilde{D})$
(b) $\widetilde{S} \subset$ Supp $\widetilde{D} \subset \rho^{-1} \nu^{-1}$ (Sing $\left.X\right)$.

Here Sing $X$ denotes the set of singular points $X$ and $\widetilde{S}$ denotes the strict 
transform of the union of 1-dimensional components of $\operatorname{Sing} X$ by $\nu \rho$.

PRoof. Let $\psi$ be the non-zero global section of $\omega_{x} \cong \mathcal{O}_{x}$. The pullback $\rho^{*} \nu^{*} \psi$ defines a 2-form with poles on $Z$. Let $-\widetilde{D}$ be the divisor defined by $\rho^{*} \nu^{*} \psi$. It is not difficult to see that $\widetilde{D}$ satisfies Conditions (a) and (b).

Q.E.D.

Corollary 2.2. $\kappa(Z)=-\infty$.

Let $\sigma: Z \rightarrow \bar{Z}$ denote the blowing-down to a minimal model.

Lemma 2.3. $\bar{Z}$ is one of the following.

( I ) a ruled surface over a curve of genus 2.

(II) a ruled surface over an elliptic curve.

(III) a minimal rational surface.

Proof. Assume that $\bar{Z}$ is not a minimal rational surface. By the classification theory of surfaces (Beauville [3]) and by Corollary 2.2, $\bar{Z}$ is a ruled surface over a curve $G$ with genus $g(G) \geqq 1$. But $G$ is the image of the strict transform $\widetilde{H}$ of $H$. We have that $0<g(G) \leqq g(\widetilde{H})$. On the other hand for each singular point $x \in H$, we have a positive integer $\delta_{x}$ depending only on the analytic structure around $x$ in $H$ such that the Plücker formula $g(\widetilde{H})+\Sigma \delta_{x}=1-\chi\left(\mathcal{O}_{H}\right)=3$ holds. Thus $g(\widetilde{H})<3$ since $H$ has a singular point on the one-dimensional singular locus of $X$. We have $g(G) \leqq 2$.

Q.E.D.

Note that that the integer $p_{a}(C)=1-\chi\left(\mathscr{O}_{c}\right)$ is called the arithmetic genus of a curve $C$.

We decompose $\sigma$ to a composition of blowing-ups of points.

$$
Z=Z_{k} \stackrel{\sigma_{k}}{\longrightarrow} Z_{k-1} \stackrel{\sigma_{k-1}}{\longrightarrow} \cdots \longrightarrow Z_{1} \stackrel{\sigma_{1}}{\longrightarrow} Z_{0}=\bar{Z} .
$$

We denote by $k$ the number of blowing-ups. We define inductively $H_{k}=\widetilde{H}$ (= strict transform of $H$ by $\nu \rho$, where $H$ is the intersection of $X$ and a general plane) $\sigma_{i}\left(H_{i}\right)=H_{i-1} \subset Z_{i-1}$ for $1 \leqq i \leqq k$, and $D_{k}=\widetilde{D}, \sigma_{i *}\left(D_{i}\right)=$ $D_{i-1}$ for $1 \leqq i \leqq k$. Let $z_{i} \in Z_{i-1}$ be the center of $\sigma_{i}$. We denote $\bar{H}=H_{0}$ and $\bar{D}=D_{0}$.

Lemma 2.4. (Umezu [27], [28]) (See Lemma 4.4). $z_{i} \in H_{i-1} \cap \operatorname{Supp} D_{i-1}$ for $1 \leqq i \leqq k$.

Lemma 2.5. We consider Case (I) and (II) in Lemma 2.3. Let $\pi: \bar{Z} \rightarrow G$ denote the projection and $\widetilde{F}$ be a general fibre of $\pi \sigma$. If $\widetilde{F} \cdot \widetilde{H}=1$, then $Z=\bar{Z}$. 
Proof. Assume $Z \neq \bar{Z}$, that is, $k \geqq 1$. Set $F=\pi^{-1} \pi\left(z_{1}\right)$. We have $F \cdot H_{0}=\widetilde{F} \cdot \widetilde{H}=1, \quad F^{2}=0$ and $F \cong P^{1}$. The strict transform $F^{\prime}$ of $F$ by $\sigma_{1}$ is an exceptional curve of the first kind which does not meet $H_{1}$. The inverse image of $F^{\prime}$ on $Z$ is an exceptional curve of the first kind which does not meet $\tilde{H}$ by Lemma 2.4. This contradicts the minimality of $\rho$.

Q.E.D.

Prட́oposition 2.6. If $\bar{Z}$ is a ruled surface over a curve $G$ with genus 2, then $X$ is a cone in $\boldsymbol{P}^{3}$ over an irreducible plane quartic curve $H$ which has only one singular point of type $A_{1}$ or $A_{2}$.

Proof. First of all note that it follows from the Hurwitz formula that $\tau=\left.\pi \sigma\right|_{\tilde{H}}: \widetilde{H} \rightarrow G$ is an isomorphism. This implies that $\widetilde{F} \cdot \widetilde{H}=1$. By Lemma 2.5 we have $Z=\bar{Z}$. Since $2=2 p_{a}(\widetilde{H})-2=\widetilde{H}^{2}-\widetilde{D} \cdot \widetilde{H}=4-\widetilde{D} \cdot \widetilde{H}$, we have $\widetilde{D} \cdot \widetilde{H}=2$. Let $\widetilde{D}=\sum_{i=1}^{n} a_{i} \widetilde{D}_{i}$ be the decomposition to irreducible components. We can assume that one of the following three cases takes place.

(1) $a_{1}=1, \widetilde{D_{1}} \cdot \widetilde{H}=2$, and $\widetilde{D}_{i} \cdot \widetilde{H}=0$ for $i \geqq 2$.

(2) $a_{1}=2, \widetilde{D}_{1} \cdot \widetilde{H}=1$, and $\widetilde{D}_{i} \cdot \widetilde{H}=0$ for $i \geqq 2$.

(3) $\quad a_{1}=a_{2}=1, \widetilde{D}_{1} \cdot \widetilde{H}=\widetilde{D}_{2} \cdot \widetilde{H}=1$, and $\widetilde{D}_{i} \cdot \widetilde{H}=0$ for $i \geqq 3$.

Now note that it follows from the Pluicker formula that $H$ has only one singular point of type $A_{1}$ or $A_{2}$ since the difference of $g(\widetilde{H})=2$ and $p_{a}(H)=3$ is 1 . Thus the union of 1-dimensional components of Sing $X$ is a line.

Assume that Case (1) takes place. Since $Z=\bar{Z}$, every fibre of $\pi$ is irreducible. The curve $\widetilde{D}_{1}$ is not contained in any fibre of $\pi$, since $\widetilde{D_{1}} \cdot \widetilde{H}=2$ and $\widetilde{F} \cdot \widetilde{H}=1$. Thus $\widetilde{F} \cdot \widetilde{D}_{1}>0$ for the general fibre $\widetilde{F}$. On the other hand $2=-2 p_{a}(\widetilde{F})+2=\widetilde{D} \cdot \widetilde{F}$. Thus $\#(\operatorname{Supp} \widetilde{D} \cap \widetilde{F})=1$ or 2 . If this number is 1 , since $\widetilde{F} \cdot \widetilde{D}_{1}>0$, we have $\#\left(\widetilde{D}_{1} \cap \widetilde{F}\right)=1, \widetilde{D} \cdot \widetilde{F}=2$ and thus $\widetilde{F}$ is tangent to $\widetilde{D}_{1}$ at one point. This is a contradiction because $\widetilde{D}_{1}$ is reduced and $\widetilde{F}$ is a general fibre. We have $\#(\operatorname{Supp} \widetilde{D} \cap \widetilde{F})=2$. Here note that $\widetilde{F}$ is sent by the morphism $\nu \rho$ isomorphically to a line in $\boldsymbol{P}^{8}$ since $\widetilde{F} \cdot \widetilde{H}=1$. It implies that $\#(\nu \rho(\widetilde{F}) \cap$ Sing $X) \geqq 2$, since $\nu \rho($ Supp $\widetilde{D}) \subset$ Sing $X$. Now, since the 1-dimensional component $\nu \rho\left(\widetilde{D}_{1}\right)$ of Sing $X$ is a line, it intersects with $\nu \rho(\widetilde{F})$ at most one point. Thus $\nu \rho(\widetilde{F})$ has to pass through an isolated singular point $P$ on $X$. Let $N$ be the plane spanned by $P$ and $\nu \rho\left(\widetilde{D}_{1}\right)$. We have $\nu \rho(\widetilde{F}) \subset N$. Thus $X \subset N$ since $\widetilde{F}$ is a general fibre. This is a contradiction. Case (1) never takes place.

Assume that Case (2) takes place. Moreover assume that $\widetilde{D}_{1} \cdot \widetilde{F}>0$. We have $\widetilde{D}_{1} \cdot \widetilde{F}=1$ since $\widetilde{D} \cdot \widetilde{F}=2$ by the adjunction formula and since $a_{1}=2$ by assumption. One sees that $\widetilde{D}_{1}$ is numerically equivalent to 
$\widetilde{H}-3 \widetilde{F}$ since $\widetilde{D_{1}} \cdot \widetilde{F}=\widetilde{D}_{1} \cdot \widetilde{H}=1$. We have $p_{a}\left(\widetilde{D}_{1}\right)=2$ by the adjunction formula. However, $\nu \rho \mid \widetilde{D}_{1}: \widetilde{D}_{1} \rightarrow \nu \rho\left(\widetilde{D}_{1}\right)=l$ is a birational morphism since $\widetilde{D}_{1} \cdot \widetilde{H}=1$, which contradicts the fact $p_{a}(l)=0$. Thus $\widetilde{D}_{1} \cdot \widetilde{F}=0$, that is, $\widetilde{D}_{1}$ is a fibre of $\pi$.

Assume that Case (3) takes place. Assume moreover that $\widetilde{D}_{i} \cdot \widetilde{F} \geqq 2$ for $i=1$ or 2 . Then $\#(\operatorname{Supp} \widetilde{D} \cap \widetilde{F})=2$ and we can deduce a contradiction as in Case (1). Thus $0 \leqq \widetilde{D}_{i} \cdot \widetilde{F} \leqq 1$. If $\widetilde{D}_{i} \cdot \widetilde{F}=1$, we can deduce a contradiction as in Case (2) as well. Thus we have $\widetilde{D}_{1} \cdot \widetilde{F}=\widetilde{D}_{2} \cdot \widetilde{F}=0$. Both $\widetilde{D}_{1}$ and $\widetilde{D}_{2}$ are fibres of $\pi$.

Now since the equalities $\widetilde{D} \cdot \widetilde{F}=2$ and $\#(\operatorname{Supp} \widetilde{D} \cap \widetilde{F})=1$ have to hold, we can write as follows.

Case (2): $\quad \widetilde{D}=2 \widetilde{D}_{1}+2 \widetilde{D}^{\prime} . \quad \widetilde{D}_{1}$ is a fibre of $\pi . \quad \widetilde{D}^{\prime}$ is a section of $\pi . \quad \widetilde{D}^{\prime} \cdot \widetilde{H}=0$. Case (3): $\quad \widetilde{D}=\widetilde{D}_{1}+\widetilde{D}_{2}+2 \widetilde{D}^{\prime} . \quad \widetilde{D}_{1}$ and $\widetilde{D}_{2}$ are fibres of $\pi$. $\widetilde{D}^{\prime}$ is a section of $\pi . \quad \widetilde{D}^{\prime} \cdot \tilde{H}=0$.

Since the ruled surface $Z=\bar{Z}$ has two disjoint sections $\tilde{H}$ and $\widetilde{D}^{\prime}$ and since $\widetilde{H}^{2}=4$, we can write it in the form $Z=\bar{Z}=\boldsymbol{P}\left(\mathcal{O}_{G} \oplus L\right)$ ( $L$ is a line bundle over $G$ with $\operatorname{deg} L=4)$ and one sees that the line bundle $\mathscr{O}_{z}(\widetilde{H})$ which defines the embedding coincides with the tautological line bundle on $\boldsymbol{P}\left(\mathscr{O}_{G} \oplus L\right)$. This fact implies our proposition.

Q.E.D.

REMARK. For the pairs $\left(X, \mathcal{O}_{X}(H)\right)$ and $\left(Z, \mathcal{O}_{Z}(\tilde{H})\right)$ in the above proposition, we can define their $\Delta$-genera $\Delta$ and degrees $d$. $\Delta$-genus is the notion due Fujita. For both pairs $\Delta=2, d=4$. However we cannot find in Fujita's theory the corresponding item to the surface discussed in this section. (Fujita [10]). This is because when $\Delta \geqq 1$, he treats only pairs $(V, M)$ where $V$ is a smooth variety and $M$ is an ample line bundle. (Needless to say, $X$ is singular and $\mathscr{O}_{z}(\tilde{H})$ is not ample.) We cannot directly generalize his theory to the case where $M$ is numerically effective.

For the pair $(V, M)$ where $V$ is a smooth irreducible surface and $M$ is an ample line bundle on $V$, we can conclude that if $\Delta(V, M)=2$, $g(V, M) \geqq 2$ and $d(V, M) \geqq 4$, then $H^{1}\left(\mathcal{O}_{V}\right)=0$. But in the case where $M$ is only numerically effective this is not the case.

Moreover it is another reason that he does not treat varieties of dimension 1 at all in [10]. The pair $(C, M)$ where $C$ is a smooth irreducible curve of genus 2 and $M$ is a line bundle of degree 4 on $C$ satisfies $\Delta=$ $g=2$ and $d=4$. If he mentions this example, it is obvious that the cone over it satisfies the same conditions.

\section{§3. The case of elliptic ruled surfaces.}

In this section we discuss the case of elliptic ruled surfaces. We 
use the same notation as in the previous section;

$$
P^{3} \supset X \stackrel{\nu}{\longleftarrow} \hat{X} \stackrel{\rho}{\longleftarrow} Z \stackrel{\sigma}{\longrightarrow} \bar{Z} \stackrel{\pi}{\longrightarrow} G, H, \tilde{H}, \bar{H}, \tilde{D}, \bar{D} \text { etc. }
$$

In this section $G$ is always a smooth irreducible elliptic curve. Note that the genus $g(\tilde{H})$ of $\tilde{H}$ satisfies $0 \leqq g(\tilde{H}) \leqq 2$ since $p_{a}(H)=3$ and $H$ has a singular point.

Case (1). $g(\tilde{H})=0$.

Note that in this case the composition $\tilde{H} \subset Z \rightarrow G$ is never surjective since $g(G)=1$. Thus $\tilde{H}$ is contained in a fibre of $Z \rightarrow G$ and we have $4=\widetilde{H}^{2} \leqq 0$, a contradiction. This case never takes place.

Case (2). $g(\tilde{H})=1$.

Note that $\operatorname{deg} N_{\widetilde{H} / Z}=\widetilde{H}^{2}=4>0$ and that $\tilde{H} \cdot A>0$ for any exceptional curve of the first kind $A$ on $Z$ by the minimality of $\rho$. It is easy to check that we can apply Proposition (1.11) in Fujita [9] even under this weaker condition. (If we apply the original proposition in [9], $\tilde{H}$ has to be ample.) Thus there is a vector bundle $E$ of $\operatorname{rank} 2$ on $G$ such that $Z=P(E)$ and $\mathcal{O}_{Z}(\tilde{H})$ coincides with the tautological line bundle on $\boldsymbol{P}(E)$. We examine $\boldsymbol{P}(E)$ more closely than in Fujita [10]. We have two cases since $\operatorname{deg} \wedge^{2} E$ is even.

(a) $E \cong M \oplus N$ for some line bundles $M$ and $N$.

(b) There is a non-splitting exact sequence $0 \rightarrow M \rightarrow E \rightarrow M \rightarrow 0$ for some line bundle $M$.

First we consider Case (a). Assume that $\operatorname{deg} M=1$. Let $G_{1} \subset \boldsymbol{P}(E)$ be the section corresponding to the projection $E=M \oplus N \rightarrow M$. Since $\mathcal{O}_{G_{1}}(\tilde{H}) \cong M$, the linear system $|\tilde{H}|$ has a fixed point on $G_{1}$, which is not the case. Thus deg $M \neq 1$ and similarly we have deg $N \neq 1$. On the other hand $\operatorname{deg} M=\widetilde{H} \cdot G_{1} \geqq 0$ and similarly $\operatorname{deg} N \geqq 0$. Since $4=\operatorname{deg} \bigcirc_{z}(\tilde{H})=$ $\operatorname{deg} \wedge^{2} E=\operatorname{deg} M+\operatorname{deg} N$, by symmetry we can assume that either (i) $(\operatorname{deg} M, \operatorname{deg} N)=(2,2)$ or (ii) $(\operatorname{deg} M, \operatorname{deg} N)=(4,0)$ holds. If the Case (ii) takes place, then obviously $X$ is a cone over a singular plane curve. In the sequel we consider the Case (i). Note that in this case the divisor $\tilde{H}$ is ample.

LEMMA 3.1. Let $M$ and $N$ be line bundles of degree 2 on a smooth elliptic curve $G$. Let $L$ be the tautological line bundle on $Z=P(M \oplus N)$. Then the linear system $|L|$ has no fixed points and it defines a morphism $\varphi_{L}: Z \rightarrow P^{3}$. The image of $\varphi_{L}$ is a quartic surface if $M$ and $N$ are not isomorphic and it is a smooth quadratic surface if $M \cong N$.

Proof. By the Riemann-Roch theorem one has $h^{\circ}(L)=h^{0}(M \oplus N)=4$. 
Let $G_{1}$ (resp. $\left.G_{2}\right)$ be the section of $\pi: P(M \oplus N) \rightarrow G$ corresponding to the projection $M \oplus N \rightarrow M$. (resp. $M \oplus N \rightarrow N$.) Since $G_{1}+\pi^{*}|M| \subset|L|$ and $G_{2}+\pi^{*}|N| \subset|L|,|L|$ has no fixed points. Here note that $\varphi=\varphi_{L}: Z \rightarrow P^{8}$ is a finite morphism since $L$ is ample.

Next assume that the image $X=\varphi(Z)$ is not a quartic surface. Then $X$ is an irreducible quadratic surface since $\operatorname{deg} X \cdot \operatorname{deg} \varphi=L^{2}=4$. The image $L_{i}=\varphi\left(G_{i}\right)$ is a line contained in $X$ for $i=1,2$, since $L \cdot G_{i}=2$ and $G_{i}$ is an elliptic curve for $i=1,2$. Note that $\phi^{-1}\left(L_{i}\right)=G_{i}$ for $i=1,2$, since $\varphi,\left.\varphi\right|_{a_{1}}$ and $\left.\varphi\right|_{a_{2}}$ are morphisms of degree 2. Thus $L_{1} \cap L_{2}=\varnothing$ since $G_{1} \cap G_{2}=\varnothing$. If $X$ is a quadratic cone, every two lines contained in it intersect. Thus $X$ is a smooth one. Then $G_{1}=\phi^{*} L_{1}$ and $G_{2}=\phi^{*} L_{2}$ are linearly equivalent because so are $L_{1}$ and $L_{2}$. It implies that $M$ and $N$ are isomorphic. Conversely assume that $M$ and $N$ are isomorphic. In this case $Z=\boldsymbol{P}^{1} \times G$ and $\operatorname{dim}\left|G_{1}\right|=1$. Note that there is a smooth elliptic curve $G^{\prime} \in\left|G_{1}\right|$ with $p \in G^{\prime}$ for any point $p \in Z$. Thus $\phi$ is never generically one-to-one, since $\left.\varphi\right|_{a^{\prime}}$ is of degree 2. One sees that the image $X=\varphi(Z)$ is a quadratic surface.

Q.E.D.

In the following we assume that $M$ and $N$ are not isomorphic. Obviously we have $L_{1} \cup L_{2} \subset \operatorname{Sing} X$, where Sing $X$ denotes the set of singular points of $X$, since $\left.\varphi\right|_{a_{i}}$ is a morphism of degree 2 . Note that every component of Sing $X$ is one-dimensional, since $\varphi$ is a finite morphism. By Lemma 2.1, we have an effective divisor $\widetilde{D} \in\left|-\omega_{z}\right|$ with Supp $\widetilde{D}=$ $(\nu \rho)^{-1}($ Sing $X) \supset G_{1} \cup G_{2}$. However since $G_{1}+G_{2} \in\left|-\omega_{z}\right|$, we have Supp $\widetilde{D}=$ $G_{1}+G_{2}$ and thus Sing $X=L_{1} \cup L_{2}$.

Let $\widetilde{F}$ denote a general fibre of $\pi: Z \rightarrow G$. We have $\left|\tilde{H}-G_{1}-G_{2}\right|=\varnothing$ since $\widetilde{F} \cdot\left(\tilde{H}-G_{1}-G_{2}\right)=-1$. It implies that $L_{1}$ and $L_{2}$ are in twisted position. The image of any member of the ruling $\boldsymbol{P}^{1}$-family is a line intersecting $L_{1}$ and $L_{2}$. Next consider $H$. The plane-section $H$ has only two singular points at $P=H \cap L_{1}$ and $Q=H \cap L_{2}$. It follows from the Plïcker formula that $P$ and $Q$ are singular points of type $A_{1}$ or $A_{2}$. Assume that either of them is of type $A_{2}$. Then $X$ is locally isomorphic to the product $\left\{\left(x, y \in C^{2}|| x|<1| y \mid,<1, x^{2}=y^{3}\right\} \times\{z \in C|| z \mid<1\}\right.$. The local generator of the dualizing sheaf $\omega_{x}$ can be identified with $d y \wedge d z / x$. Setting $x=t^{8}$ and $y=t^{2}$, one sees that its pull-back $2 d t \wedge d z / t^{2}$ has a multiple pole on $Z$. But $\widetilde{D}=G_{1}+G_{2}$ has no multiple component. Thus both $P$ and $Q$ are of type $A_{1}$.

Secondly we consider the Case (b). The vector bundle $E$ of rank 2 is uniquely determined by $M$ up to isomorphisms. Note that $4=$ $\operatorname{deg} \mathcal{O}_{z}(\widetilde{H})=\operatorname{deg} \wedge^{2} E=2 \operatorname{deg} M$. 
Lemma 3.2. Let $M$ be a line bundle of degree 2 on a smooth elliptic curve $G$ and let $E$ be the vector bundle which has the non-splitting exact sequence $0 \rightarrow M \rightarrow E \rightarrow M \rightarrow 0$. Then the linear system $|L|$ defined by the tautological line bundle $L$ on $Z=\boldsymbol{P}(E)$ has no fixed points and it defines a morphism $\varphi_{L}: Z \rightarrow P^{3}$. The image of $\varphi_{L}$ is a quartic surface.

Proof. One has easily $h^{0}(L)=h^{0}(E)=2 h^{0}(M)=4$. Let $G_{1}$ be the section of $\pi: Z=P(E) \rightarrow G$ associated with the above exact sequence. Since $G_{1}+\pi^{*}|M| \subset|L|,|L|$ has no fixed points outside $G_{1}$. Next consider the exact sequence $0 \rightarrow \mathcal{O}_{Z}\left(L-G_{1}\right) \rightarrow \mathcal{O}_{Z}(L) \rightarrow \mathcal{O}_{G_{1}}(L) \rightarrow 0$. Since $H^{1}\left(\mathcal{O}_{Z}\left(L-G_{1}\right)\right) \cong$ $H^{1}\left(\mathcal{O}_{G}(M)\right)=0$ and since the linear system $\left|\mathcal{O}_{G_{1}}(L)\right| \cong|M|$ has no fixed points, one can conclude that $|L|$ has no fixed points on $G_{1}$, either. Thus we have a finite morphism $\varphi=\varphi_{L}: Z \rightarrow \boldsymbol{P}^{3}$.

Next assume that the image $X=\varphi(Z)$ is not a quartic surface. Then $X$ is an irreducible quadratic surface and the image $L_{1}=\varphi\left(G_{1}\right)$ is a line contained in $X$. Assume moreover that $X$ has a singular point. Let $L_{2}$ be another line contained in $X$. Then $2 L_{1}$ and $L_{1}+L_{2}$ are Cartier divisors on $X$ and they are linearly equivalent. Note that $\phi^{-1}\left(L_{i}\right)=G_{i}$ as sets. Thus we have $\phi^{*}\left(2 L_{1}\right)=2 G_{1}$ and $\phi^{*}\left(L_{1}+L_{2}\right)=G_{1}+G_{2}$ as divisors, where $G_{2}$ is a divisor different from $G_{1}$. It implies that $\operatorname{dim}\left|G_{1}\right| \geqq 1$ since $G_{2} \in\left|G_{1}\right|$. It contradicts the fact that the exact sequence in the lemma does not split. Next assume that $X$ is smooth. Let $L_{2}$ be another line contained in $X$ with $L_{1} \cap L_{2}=\varnothing$. We have $G_{2}=\varphi^{*} L_{2} \in\left|G_{1}\right|$, a contradiction in this case, too. Thus $X$ is a quartic surface.

Q.E.D.

Now we can conclude Sing $X=\varphi\left(G_{1}\right)$ is a line by the same reasoning as that in the Case (a) since $2 G_{1} \in\left|-\omega_{z}\right|$. Moreover it is easy to see that the plane section $H$ has an $A_{3}$-singular point at $H \cap \varphi\left(G_{1}\right)$. Case (3). $g(\widetilde{H})=2$.

In this case the plane-section $H$ has a unique singular point since $p_{a}(H)-g(\widetilde{H})=1$. It imples that the union of one-dimensional components of Sing $X$ coincides with a line $l_{0}$. Let $P \subset P^{3}$ be a general plane containing $l_{0}$. One of the following two cases takes place since $X$ is reduced. (a) $X \cap P=2 l_{0}+C$, where $C$ is an irreducible conic.

(b) $X \cap P=2 l_{0}+l_{1}+l_{2}$, where $l_{1}$ and $l_{2}$ are two lines intersecting at one point.

Assume that the Case (a) takes place. In this case $C$ is rational and thus it is a fibre of $Z \rightarrow G$. Since $G$ is elliptic, we have $\operatorname{dim}|C|=0$. However, on the other hand, since $P$ is a general plane, we have $\operatorname{dim}|C| \geqq 1$, which is a contradiction. Next assume that the Case (b) takes place. Obviously $\widetilde{H} \cdot \widetilde{l}_{i}=1$ for $i=1,2$ where $\widetilde{l}_{i}$ denotes the strict transform 
of $l_{i}$ in $Z$. Since $\tilde{l}_{1}$ coincides with a fibre of $Z \rightarrow G, \tilde{H}$ is a section of $Z \rightarrow G$. We have $2=g(\widetilde{H})=g(G)=1$, a contradiction. Thus the case $g(\tilde{H})=2$ does not take place.

\section{§4. The case of rational surfaces.}

In this section we deal with the case where $Z$ is rational. We use the notion of 4 -genera due to Fujita (Fujita [8], [9], [10]) as the main tool. Now recall that the integer $\Delta(V, M)=\operatorname{dim} V+\operatorname{deg} M-h^{0}(M)$ is the $\Delta$-genus of an irreducible reduced (possibly singular) varity $V$ and a line bundle $M$ on $V$. Let $\nu: \hat{X} \rightarrow X$ denote the normalization of $X$ and $\hat{L}=$ $\nu^{*} \mathcal{O}_{p s}(1) . \quad \hat{L}$ is ample. We consider the integer $\Delta=\Delta(\hat{X}, \hat{L})$. By definition $\Delta \leqq 2$. On the other hand it is known that $\Delta$ is non-negative. (Fujita [8])

Case (1). $\Delta=0$.

There is a complete list of pairs $(V, M)$ such that $M$ is ample and $\Delta(V, M)=0$. (Fujita [8]). Thus we have only to pick up items corresponding to surfaces of degree 4 . We have three cases. In any case $\hat{L}$ is very ample and thus $\hat{X}$ is a surface in $P^{s}$

(a) $(\hat{X}, \hat{L})=\left(P^{2}, \mathcal{O}_{\mathbf{P}^{2}}(2)\right)$.

(b) $\hat{X}=\boldsymbol{P}\left(\mathcal{O}_{\mathbf{P}_{1}}(a) \oplus \mathcal{O}_{\mathbf{P}_{1}}(b)\right)$ and $\hat{L}$ is the tautological line bundle on $P\left(\mathcal{O}_{\mathbf{P} 1}(a) \oplus \mathcal{O}_{\mathbf{P}^{1}}(b)\right)$ where $(a, b)=(2,2)$ or $(3,1)$.

(c) Let $P^{4} \subset P^{5}$ be a hyperplane and $G \subset P^{4}$ be the image of $P^{1}$ by the morphism defined by $\left|\mathscr{O}_{F^{1}}(4)\right| . \quad \hat{X}$ is the cone over $G$ with vertex $v$ where $v \in P^{5}$ is a point outside $\boldsymbol{P}^{4} . \hat{L}=\left.\mathcal{O}_{P^{5}}(1)\right|_{\hat{x}}$.

Note that one can conclude by the definition of $L$ that $X$ is an image by a projection $\boldsymbol{P}^{5} \ldots \rightarrow \boldsymbol{P}^{s}$ whose center is a line disjoint from $\hat{X}$. Thus we have the results in this case.

Case (2). $\quad \Delta=1$.

When $\Delta \geqq 1$, only pairs $(V, M)$ where $V$ is a smooth irreducible variety and $M$ is an ample line bundle were treated in Fujita's theory.

Let $\rho: Z \rightarrow \hat{X}$ be the minimal resoultion and let $\widetilde{H}$ be the strict transform on $Z$ of the general plane section. Since $\hat{X}$ is normal, we have $\rho_{*} \mathcal{O}_{Z}(\widetilde{H})=\hat{L}$ and $\Delta\left(Z, \mathcal{O}_{Z}(\widetilde{H})\right)=\Delta(\hat{X}, \hat{L})=1$. Fujita's theory can be applied to neither $\left(Z, \mathcal{O}_{Z}(\widetilde{H})\right)$ nor $(\hat{X}, \hat{L})$. But only by making his arguments slightly precise we can reach the conclusion in our case. Now $H^{0}\left(\mathcal{O}_{Z}(\widetilde{H})\right) \rightarrow$ $H^{0}\left(\mathcal{O}_{\widetilde{H}}(\widetilde{H})\right)$ is surjective since $H^{1}\left(\mathcal{O}_{Z}\right)=0$. It implies that $\Delta\left(\widetilde{H}, \mathscr{O}_{\widetilde{H}}(\widetilde{H})\right)=$ $\Delta\left(Z, \mathcal{O}_{Z}(\widetilde{H})\right)=1$. On the other hand we have $\Delta\left(\widetilde{H}, \mathcal{O}_{\widetilde{H}}(\widetilde{H})\right)=g(\widetilde{H})-$ $h^{0}\left(\omega_{\widetilde{H}}(-\widetilde{H})\right)$ by Riemann-Roch. Note that $\operatorname{deg} \omega_{\widetilde{H}}(-\widetilde{H})=2 g(\widetilde{H})-2-4<0$ since $g(\widetilde{H}) \leqq 2$ in our case. Thus $g(\widetilde{H})=\Delta\left(\widetilde{H}, \widehat{O}_{\widetilde{H}}(\widetilde{H})\right)=1$. Next we consider the exact sequence $0 \rightarrow \omega_{Z} \rightarrow \omega_{Z}(\widetilde{H}) \rightarrow \omega_{\tilde{H}} \rightarrow 0$. We have $h^{0}\left(\omega_{Z}(\widetilde{H})\right)=h^{0}\left(\omega_{\tilde{H}}\right)=1$, 
since $h^{0}\left(\omega_{z}\right)=h^{1}\left(\omega_{z}\right)=0$. Let $F$ be the unique element in the linear system $\left|\omega_{z}+\tilde{H}\right|$. Assume that $F$ is non-zero. We will deduce a contradiction. By the adjunction formula $F \cdot \tilde{H}=\left(\omega_{z}+\tilde{H}\right) \cdot \tilde{H}=2 g(\tilde{H})-2=0$. Thus by the Hodge index theorem we have $F^{2}<0$. Let $F=\sum a_{i} F_{i}$ be the irreducible decomposition. There is a index $i$ with $F_{i} \cdot F<0$. Note that $F_{i} \cdot \widetilde{H} \geqq 0$ since $\widetilde{H}$ is numerically effective and that $2 p_{a}\left(F_{i}\right)-2-F_{i}^{2} \geqq 0$. (Otherwise $p_{a}\left(F_{i}\right)=0$ and $F_{i}^{2}=-1$ since $F_{i}^{2}<0$. It implies that $F_{i}$ is an exceptional curve of the first kind. It contradicts the fact that $\rho$ is the minimal resolution since $F_{i} \cdot \tilde{H}=0$.) Thus we have $0>F_{i} \cdot F=F_{i} \cdot\left(\omega_{z}+\tilde{H}\right)=2 p_{a}\left(F_{i}\right)-2-F_{i}^{2}+F_{i} \cdot \tilde{H} \geqq 0$, a contradiction. One sees that $F=0$ and $\mathscr{O}_{z}(\tilde{H})=-\omega_{z}$. Thus $Z$ is a generalized Del Pezzo surface and by Demazure [6] we obtain the conclusion in section 1 in this case.

REMARK. Note that in this case the one-dimensionl singular locus on $X$ is a quadratic curve or a double line, since the difference of $p_{a}(H)$ and $g(\tilde{H})$ is 2. Thus this is the case considered closely by Segre in [23]. He showed first that our surface is a projection of the complete intersection of two hypersurfaces of degree 2 in $P^{4}$. Then by considering normal forms of two quadratic forms with 5 variables and by classifying the position of the central point of the projection, he gave a very fine list about the combination of singularities on the surface and other properties.

Case (3). $\quad \Delta=2$.

We use the same notations as above. By similar arguments as in the beginning of Case (2), one sees $g(\tilde{H})=2$. One-dimensional singular locus is a line, since $p_{a}(H)-g(\tilde{H})=1$. Consider the exact sequence $0 \rightarrow \omega_{z} \rightarrow$ $\omega_{Z}(\tilde{H}) \rightarrow \omega_{\tilde{H}} \rightarrow 0$. We have $h^{0}\left(\omega_{Z}\right)=h^{1}\left(\omega_{Z}\right)=0$ since $Z$ is rational. It implies that $h^{0}\left(\omega_{z}(\tilde{H})\right)=h^{0}\left(\omega_{\widetilde{H}}\right)=2$. Let $\widetilde{F}+\Delta \in\left|\omega_{z}+\widetilde{H}\right|$ be a general member, where $\widetilde{F}$ is the moving part and $\Delta$ is the fixed part. Note that $\Delta \cdot \widetilde{H}=0$ since the linear system $\left|\omega_{\tilde{H}}\right|$ has no fixed points. Thus $\widetilde{F} \cdot \tilde{H}=(\widetilde{F}+\Delta) \cdot \tilde{H}=$ $\left(\omega_{Z}+\tilde{H}\right) \cdot \tilde{H}=2 g(\tilde{H})-2=2$. The rational map $\varphi=\varphi_{\omega_{Z}+\widetilde{H}}: Z \cdots \rightarrow P^{1}$ associated with $\omega_{Z}+\tilde{H}$ is defined. Let $\mu: Z^{\prime} \rightarrow Z$ be a composition of blowing-ups such that the composition $\psi=\varphi \mu: Z^{\prime} \rightarrow P^{1}$ is defined as a morphism. We assume moreover that $\mu$ is minimum among such compositions of blowingups. Let $F^{\prime}$ be the strict transform of $\widetilde{F}$ by $\mu$. This $F^{\prime}$ is a general fibre of $\psi$ and it is smooth. Let $H^{\prime}$ be the total transform of $\tilde{H}$ by $\mu$. Note that $F^{\prime} \cdot H^{\prime}=F^{\prime} \cdot \mu^{*} \widetilde{H}=\mu_{*} F^{\prime} \cdot \widetilde{H}=\widetilde{F} \cdot \hat{H}=2$.

LEMMA 4.1. $F^{\prime}$ is irreducible and is isomorphic to $\boldsymbol{P}^{1}$.

Proof. Assume that $F^{\prime}$ is reducible. $F^{\prime}$ has two or more connected components since it is smooth. We have the Stein factorization of $\psi$ in 
the following form: Each of fibre of the morphism $\beta: Z^{\prime} \rightarrow G$ is connected. The morphism $\alpha: G \rightarrow P^{1}$ is a finite one with $\operatorname{deg} \alpha=a \geqq 2$ and $\psi=\alpha \beta$. Moreover $G$ is smooth.

Since $H^{0}\left(\Omega_{Z^{\prime}}^{1}\right)=0, G$ is rational. We have $2=h^{0}\left(\mathcal{O}_{Z^{\prime}}\left(F^{\prime}\right)\right)=h^{0}\left(\mathcal{O}_{G}(a)\right) \geqq 3$, a contradiction. Thus $F^{\prime}$ is irreducible.

Next assume that $g\left(F^{\prime}\right) \geqq 1$. The restriction $\left.\nu \rho \mu\right|_{F^{\prime}}$ is a morphism of degree 2 , since $F^{\prime} \cdot H^{\prime}=2$. Since members in $\left|F^{\prime}\right|$ cover $Z^{\prime}$, $\nu \rho \mu$ is never generically one-to-one, which is a contradiction. Thus $g\left(F^{\prime}\right)=0$. Q.E.D.

Now we give a remark here.

If we can assume that $\widetilde{H}$ is ample, we can show that $B s\left|\omega_{z}+\widetilde{H}\right|=\varnothing$ i.e., the linear system $\left|\omega_{z}+\widetilde{H}\right|$ has no base points, in the following way. First of all we have the fixed component $\Delta=0$ since $\Delta \cdot \widetilde{H}=0$. Thus $B s\left|\omega_{z}+\tilde{H}\right|$ is at most finite points. Let $z_{1}, \cdots, z_{k}$ be all points in $B s\left|\omega_{z}+\widetilde{H}\right|$ including infinitely near points. Let $m_{i}$ be the multiplicity of a general member $\widetilde{F} \in\left|\omega_{z}+\tilde{H}\right|$ at $z_{i}$ for $1 \leqq i \leqq k$. We have $p_{a}(\widetilde{F})-\sum m_{i}\left(m_{i}-1\right) / 2=p_{a}\left(F^{\prime}\right)=0$. On the other hand $p_{a}\left(\widetilde{F^{*}}\right)=\left(\widetilde{F}^{2}+\omega_{z} \cdot \widetilde{F}\right) /$ $2+1=\widetilde{F}^{2}-\widetilde{H} \cdot \widetilde{F} / 2+1=\widetilde{F}^{2}=\widetilde{F}^{\prime 2}+\sum m_{i}^{2}=\sum m_{i}^{2}$. They imply that $0<$ $\sum_{i=1}^{k} m_{i}\left(m_{i}+1\right)=0$, a contradiction, if $k \geqq 1$. Thus $k=0$ and $B s\left|\omega_{z}+\widetilde{H}\right|=\varnothing$.

However, in our case we can assume only that $\tilde{H}$ is numerically effective. Therefore we need more precise arguments.

LEMMA 4.2. Let $f: M \rightarrow G$ be a proper morphism from a smooth surface $M$ to a smooth curve $G$. Assume that there is a point $x \in G$ such that the fibre $f^{-1}(x)$ is isomorphic to $\boldsymbol{P}^{1}$. Then any singular fibre of $f$ contains an exceptional curve of the first kind. Moreover let $\sigma: M \rightarrow M^{\prime}$ denote the contraction of any exceptional curve of the first kind $E$ such that $f(E)$ is one point. Then there is a morphism $f^{\prime}: M^{\prime} \rightarrow G$ with $f=f^{\prime} \sigma$.

Proof. This is an easy consequence of the adjunction formula and the Hartogs' extension theorem.

Q.E.D.

Applying Lemma 4.2 repeatedly, we have the next commutative diagram.

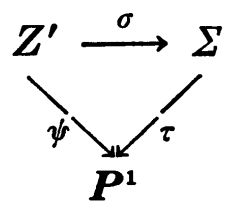

The morphism $\sigma$ is a birational one and $\tau$ has no singular fibre. By the classification theory of rational surfaces (Beauville [3]), one sees that $\Sigma$ 
coincides with the Hirzebruch surface $\Sigma_{m}$ for some $m \geqq 0$. (See Section 1.) Set $\bar{H}=\sigma_{*} H^{\prime}$. By $\bar{F}$ we denote a general fibre of $\tau$. Note that $\bar{F} \cdot \bar{H}=\bar{F} \cdot \sigma_{*} H^{\prime}=\sigma^{*} \bar{F} \cdot H^{\prime}=F^{\prime} \cdot H^{\prime}=2$.

LEMMA 4.3. Let $E \subset Z^{\prime}$ be an exceptional curve of the first kind such that $\psi(E)$ is one point. Then $E \cdot H^{\prime}>0$.

Proof. Assume that there is an exceptional curve of the first kind $E \subset Z^{\prime}$ such that $\psi(E)$ is one point and $E \cdot H^{\prime}=0$. By the minimumness of $\mu, \mu_{*} E \neq 0$. However $\mu_{*} E \cdot \tilde{H}=E \cdot \mu^{*} \tilde{H}=E \cdot H^{\prime}=0$. Thus by the Hodge index theorem $\left(\mu_{*} E\right)^{2}<0$. On the other hand $-1=E^{2} \leqq\left(\mu_{*} E\right)^{2}$. Namely we have $\left(\mu_{*} E\right)^{2}=-1$ and $\mu_{*} E$ is an exceptional curve of the first kind on $Z$. It follows from the minimality of $\rho$ that $E \cdot H^{\prime}=\mu_{*} E \cdot \tilde{H}>0$, which is a contradiction.

Q.E.D.

LEMMA 4.4. We write $\sigma: Z^{\prime} \rightarrow \Sigma$ as a composition of $k$ blowing-ups.

$$
Z^{\prime}=Z_{k} \stackrel{\sigma_{k}}{\longrightarrow} Z_{k-1} \stackrel{\sigma_{k-1}}{\longrightarrow} \cdots \longrightarrow Z_{1} \stackrel{\sigma_{1}}{\longrightarrow} Z_{0}=\Sigma \text {. }
$$

We define inductively $H_{k}=H^{\prime}$ and $\sigma_{i}\left(H_{i}\right)=H_{i-1}$ for $k \geqq i \geqq 1$. Let $z_{i} \in Z_{i-1}$ be the center of $\sigma_{i}$.

(1) $z_{i} \in H_{i-1}$ for $1 \leqq i \leqq k$.

(2) $H_{0}=\bar{H}$ is smooth.

Proof. Assume that (1) does not hold. Set $a=\max \left\{i \mid z_{i} \notin H_{i-1}\right.$, $1 \leqq i \leqq k\}$. Then $E=\left(\sigma_{a} \sigma_{a+1} \cdots \sigma_{k}\right)^{-1}\left(z_{a}\right)$ is an exceptional curve of the first kind which does not meet $H^{\prime}$. We have a contradiction by Lemma 4.3 since $\psi(E)$ is a point. Thus (1) holds.

Next assume that $z \in \bar{H}$ is a singular point with multiplicity $r$. We will deduce a contradiction. Since $\widetilde{H}$ is non-singular, we have $Z^{\prime} \neq \Sigma$. Set $\bar{F}_{z}=\tau^{-1}(\tau(z))$. The inequality $r \leqq \bar{F}_{z} \cdot \bar{H}=\bar{F} \cdot \bar{H}=2$ holds. Thus $r=2$ and $\bar{F}_{z}$ does not meet $\bar{H}$ outside $z$. By exchanging the order of blowingups, we can assume the center $z_{1}$ of $\sigma_{1}$ coincides with $z$. Let $F_{1}$ denote the strict inverse image of $\bar{F}_{z}$ by $\sigma_{1}$. $F_{1}$ is an exceptional curve of the first kind which does not meet $H_{1}$. By $(1),\left(\sigma_{2} \sigma_{3} \cdots \sigma_{k}\right)^{-1}\left(F_{1}\right)$ is an exceptional curve of the first kind which does not meet $H^{\prime}$. Thus by Lemma 4.3 we obtain a contradiction. Q.E.D.
Q

Let $\bar{C}$ (resp. $\bar{B}$ ) denote a section of $\tau: \Sigma=\Sigma_{m} \rightarrow \boldsymbol{P}^{1}$ with $\bar{C}^{2}=m$ (resp. $\left.\bar{B}^{2}=-m\right)$. We have integers $a, b$ with $\bar{H} \sim a \bar{C}+b \bar{F}$ since $\operatorname{Pic}\left(\Sigma_{m}\right)=$ $Z[\bar{C}]+Z[\bar{F}]$. Here $a=\bar{H} \cdot \bar{F}=2$ and $b=\bar{H} \cdot \bar{B} \geqq 0$. On the other hand it is easy to see $\omega_{\Sigma_{m}} \sim-2 \bar{C}+(m-2) \bar{F}$. By Lemma 4.4 (2), one has 


$$
2=2 g(\bar{H})-2=\left(\bar{H}+\omega_{\Sigma_{m}}\right) \cdot \bar{H}=(b+m-2) \bar{F} \cdot(2 \bar{C}+b \bar{F})=2(b+m-2) .
$$

Thus we have the equality $\left({ }^{*}\right) b+m=3$. This equality and Lemma 4.4 yield strong conclusions. First we can show that $Z=Z^{\prime}$ and that $B s\left|\omega_{z}+\widetilde{H}\right|=\varnothing$.

Indeed Lemma 4.4 implies $\omega_{z^{\prime}}+H^{\prime} \sim \sigma^{*}\left(\omega_{\Sigma_{m}}+\bar{H}\right)$. By the above equality $\left(^{*}\right) \omega_{\Sigma_{m}}+\bar{H} \sim \bar{F}$ and thus one has $\omega_{z^{\prime}}+H^{\prime} \sim \sigma^{*} \bar{F}=F^{\prime}$. Assume that $Z \neq Z^{\prime}$. In this case there is an exceptional curve $E$ of the first kind on $Z^{\prime}$ such that $H^{\prime} \cdot E=0$. We have $-1=\omega_{Z^{\prime}} \cdot E=\left(\omega_{Z^{\prime}}+H^{\prime}\right) \cdot E=$ $F^{\prime} \cdot E \geqq 0$, a contradiction. Thus $Z^{\prime}=Z$. In particular $\widetilde{F}=F^{\prime} \in\left|\omega_{Z^{\prime}}+H^{\prime}\right|=$ $\left|\omega_{z}+\widetilde{H}\right|$ and $\widetilde{F}^{2}=0$. By Lemma 4.1 we have $\widetilde{F} \cong P^{1}$ and thus $B s\left|\omega_{z}+\tilde{H}\right|=\varnothing$.

Proposition. 4.5. One of the following holds.

(C-1) There is a composition of 9 blowing-ups $\sigma: Z \rightarrow P^{2}$ such that $\tilde{H} \sim$ $4 E_{0}-2 E_{1}-\sum_{i=2}^{\theta} E_{i}$, where $E_{0}$ is the total transform of a line in $P^{2}$ by $\sigma$. (C-2) There is a composition of 8 blowing-ups $\sigma: Z \rightarrow \Sigma_{2}$ such that $\tilde{H} \sim$ $2 \sigma^{*} \bar{C}+\sigma^{*} \bar{F}-\sum_{i=1}^{8} E_{i}$, where $\bar{C}$ denote a section of $\tau: \Sigma_{2} \rightarrow \boldsymbol{P}^{1}$ with $\bar{C}^{2}=2$ and $\bar{F}$ is a fibre of $\tau$.

Here in both cases $E_{i}$ denotes the total transform of the exceptional curve of the $i$-th blowing-up for $i \geqq 1$.

Furthermore the linear system $\left|\omega_{z}+\widetilde{H}\right|$ contains an element $\widetilde{F}$ with $\widetilde{F} \cong \boldsymbol{P}^{1}, \widetilde{F}^{2}=0$ and $\widetilde{F} \cdot \widetilde{H}=2$.

Now a birational morphism $f: Z \rightarrow \Sigma_{m}$ to a Hirzebruch surface is called an admissible blowing-down, if the composition $\tau f: Z \rightarrow P^{1}$ coincides with the morphism given by the linear system $\left|\omega_{Z}+\widetilde{H}\right|$. The composition $Z \rightarrow P^{2}$ of an admissible blowing-down $Z \rightarrow \Sigma_{1}$ and the blowing-down $\Sigma_{1} \rightarrow$ $\boldsymbol{P}^{2}$ is also called an admissible blowing-down to $\boldsymbol{P}^{2}$.

Proof. The latter half has been verified in the above.

We consider the admissible blowing-down $\sigma: Z=Z^{\prime} \rightarrow \Sigma_{m}$. Now we have that $\bar{H}^{2}=4 m+4(3-m)=12$, since $\bar{H} \sim 2 \bar{C}+(3-m) \bar{F}$. Thus in view of Lemma 4.4 the number of the blowing-ups between $Z$ and $\Sigma_{m}$ coincides with $k=\bar{H}^{2}-\widetilde{H}^{2}=12-4=8$. On the other hand one sees that $m=0,1,2$ or 3 , since $b+m=3, b \geqq 0$ and $m \geqq 0$. It is easy to see that if there is an admissible blowing-down $Z \rightarrow \Sigma_{0}$, then there is another admissible blowing-down $Z \rightarrow \Sigma_{1}$. If there is an admissible blowing-down $\sigma: Z \rightarrow \Sigma_{1}$, then composing the blowing-down $\Sigma_{1} \rightarrow P^{2}$, we have the blowing-down $\sigma: Z \rightarrow P^{2}$. Since $\tilde{H} \sim 2 \bar{\sigma}^{*} \bar{C}+2 \bar{\sigma}^{*} \bar{F}-\sum_{t=2}^{9} E_{t}$, we have the above expression of $\tilde{H}$. If we have an admissible blowing-down $Z \rightarrow \Sigma_{3}$, then $b=0$ and $\bar{H} \sim 2 \bar{C}$. In particular $\bar{H} \cap \bar{B}=\varnothing$ since $\bar{H} \cdot \bar{B}=2 \bar{C} \cdot \bar{B}=0$. Now the center 
$z_{1}$ of the first blowing-up lies on $\bar{H}$ by Lemma 4.4 (1). Thus $z_{1} \notin \bar{B}$. By using this fact, it is easy to see that there is another admissible blowingdown $Z \rightarrow \Sigma_{2}$. The expression of $\tilde{H}$ is easily obtained since $b=1$. Q.E.D.

REMARK. In Section 5 we show that if case (C-2) takes place, then there is another blowing-down to $P^{2}$ satisfying (C-1).

Proposition 4.6. $\operatorname{dim}\left|-\omega_{z}\right|=0$.

Proof. There is an element $\widetilde{D} \in\left|-\omega_{z}\right|$ by Lemma 2.1. Let $\widetilde{F}$ be the divisor in Proposition 4.5. Note that $\widetilde{H} \sim \widetilde{F}+\widetilde{D}$. We consider the next exact sequence. $\quad 0 \rightarrow \mathcal{O}_{Z}(\widetilde{D}) \rightarrow \mathcal{O}_{Z}(\widetilde{H}) \rightarrow \mathcal{O}_{\widetilde{F}}(\tilde{H}) \rightarrow 0$.

Assume that $\operatorname{dim}\left|-\omega_{Z}\right| \geqq 1$. We have $h^{0}\left(\mathcal{O}_{Z}(\widetilde{D})\right)=\operatorname{dim}\left|-\omega_{Z}\right|+1 \geqq 2$. Since $h^{2}\left(\mathcal{O}_{Z}(\widetilde{D})\right)=h^{0}\left(\mathcal{O}_{Z}(-2 \widetilde{D})\right)=0$ and since $\chi\left(\mathcal{O}_{Z}(\widetilde{D})\right)=\left(\widetilde{D}^{2}+\widetilde{D}^{2}\right) / 2+\chi\left(\mathscr{O}_{z}\right)=$ 1 , one sees that $h^{1}\left(\mathscr{C}_{z}(\widetilde{D})\right) \geqq 1$. On the other hand it follows from the Riemann-Roch formula that $H^{1}\left(\mathcal{O}_{Z}(\tilde{H})\right)=0$ since $h^{0}\left(\mathcal{O}_{Z}(\tilde{H})\right)=4$ and $h^{2}\left(\mathscr{O}_{Z}(\tilde{H})\right)=$ $h^{0}\left(\mathcal{O}_{Z}(-\tilde{H}-\widetilde{D})\right)=0$. Thus $H^{0}\left(\mathcal{O}_{Z}(\widetilde{H})\right) \rightarrow H^{0}\left(\mathcal{O}_{\widetilde{F}}(\widetilde{H})\right) \cong H^{0}\left(\mathcal{O}_{\mathbf{P} 1}(2)\right) \cong C^{3}$ is not surjective. It implies that $\nu \rho(\widetilde{F})$ is a line and that $\left.\nu \rho\right|_{\tilde{F}}$ is a morphism of degree 2. The members in $|\widetilde{F}|$ cover $Z$, and $\nu \rho$ is never generically one-to-one, which is a contradiction.

Q.E.D.

REMARK. If we see the item in Fujita [10] corresponding to the case dimension $=2, \Delta=2$, sectional genus $(=g(\widetilde{H})$, in our case $)=2$ and degree $=4$, it says only "the normalization of singular hypersurface" or "hyperelliptic". There is no description such that it is rational or such that it can be obtained by 9 blowing-ups from $\boldsymbol{P}^{2}$. However, we can guess from his description in the case degree $\geqq 5$ that he knows that (C-1) in Proposition 4.5 holds in the case where he treats, i.e., in the case $Z=\hat{X}$ in our notation.

Now it should be remarked here that in [18] Kummer gave the classification of quartic surfaces in $\boldsymbol{P}^{3}$ containing infinitely many quadratic curves. He showed the existence of the following cases: surfaces with a double irreducible quadratic curve or a double line; ruled quartic surfaces; the surfaces $F^{2}=A B C D$ where $F=0$ is a quadratic surface and $A, B, C, D$ coaxal planes; Steiner's surface.

According to Jessop [12] to these surfaces discussed by Kummer the surface with the following equation must be added:

$$
\{x w+F(y, z, w)\}^{2}=G(z, w)^{4}
$$

where $F$ is a homogeneous polynomial of degree 2 and $G$ is of degree 1 . (See Umezu [28]. She discovered again some of above Kummer's surfaces from her view-point.) 
In [19] Nöther showed that there are only three classes of rational quartic surfaces with only isolated singular points. (Two classes of surfaces with either the singularity $\widetilde{E}_{8}$ or its degenerated singularity, one classes of surfaces with either the singularity $\widetilde{E}_{7}$ or its degenerated singularity.)

\section{§5. Rational surfaces in case (C).}

In this section we study properties of surfaces in Case (C-1) and (C-2) in Proposition 4.5. We use the same notations as in previous sections such as $X, \hat{X}, Z, \nu, \rho, \sigma, P^{2}, \Sigma_{2}, \widetilde{D}, \bar{D}, H, \tilde{H}, \bar{H}, \bar{C}, \bar{F}, \widetilde{C}, \widetilde{F}$ etc. In particular $\widetilde{F}$ is a divisor on $Z$ such that $\widetilde{F} \cong P^{1}, \widetilde{F}^{2}=0, \widetilde{F} \cdot \widetilde{D}=2$ and $\widetilde{H} \sim \widetilde{D}+\widetilde{F}$. In Case (C-1) $\widetilde{F} \sim \widetilde{E}_{0}-\widetilde{E}_{1}$ and in Case $(C-2) \quad \widetilde{F}=\sigma^{*} \bar{F}$. Recall that $\operatorname{dim}|\widetilde{D}|=0$ if $Z$ corresponds to a quartic surface $X$.

LEMma 5.1. For any non-zero effective divisors $P$ and $Q$ on $Z$ such that $\widetilde{D}=P+Q$, the inequality $P \cdot Q \geqq 2$ holds.

Proof. Obviously $H^{0}\left(\mathcal{O}_{Z}(-P)\right)=0$ and $H^{2}\left(\mathcal{O}_{Z}(-P)\right) \cong H^{0}\left(\mathcal{O}_{Z}(P-\widetilde{D})\right)=$ $H^{0}\left(\mathcal{O}_{Z}(-Q)\right)=0$. Thus by the Riemann-Roch theorem

$$
0 \geqq \chi\left(\mathcal{O}_{Z}(-P)\right)=\left(P^{2}-P \cdot(P+Q)\right) / 2+1=-P \cdot Q / 2+1 \text {. }
$$

LemMA 5.2. We set $\widetilde{D}=\sum_{i=1}^{r} a_{i} \widetilde{D}_{i}$ where $\widetilde{D}_{i}^{\prime}$ 's are mutually different reduced irreducible curves and $a_{i}^{\prime}$ 's are positive integers. The number of different components of $\widetilde{D}$ is $r$.

(0) Note that $\widetilde{H} \cdot \widetilde{D}_{i}=\widetilde{D} \cdot \widetilde{D}_{i}+\widetilde{F} \cdot \widetilde{D}_{i}$ for every $i$.

(1) If $r=1$, then $a_{1}=1$ and $p_{a}\left(\widetilde{D}_{1}\right)=1$.

Assume that $r \geqq 2$.

(2) Every irreducible component $\widetilde{D}_{i}$ is a smooth rational curve and $\widetilde{D} \cdot \widetilde{D}_{i}=2+\widetilde{D}_{i}^{2}$ for every $i$.

(3) $\widetilde{D}_{i}^{2}=-1,-2$ or -3 for every $i$.

(4) Set $N(-t)=\sum a_{j}$ where summation runs over the set of indices $j$ with $\widetilde{D}_{j}^{2}=-t$. Then $N(-1)=N(-3)=N$ and this common value $N$ is either 0,1 , or 2.

(5) If $\widetilde{F} \cdot \widetilde{D}_{i}=0$ for some $i$, then $\widetilde{D}_{i}^{2}=-1$ or -2 .

(6) If $\tilde{H} \cdot \widetilde{D}_{i}=0$ for some $i$, then $\widetilde{D}_{i}^{2}=-2$ or -3 .

Proof. (1) Since $\omega_{Z}^{2}=0 \neq 8,9, Z$ is not a minimal model. Thus there is an exceptional curve $E \subset Z$ of the first kind. We have $1=E \cdot \widetilde{D}=$ $a_{1} E \cdot \widetilde{D}_{1}$. Thus $a_{1}=1$. By the adjunction formula we have $p_{a}\left(\widetilde{D}_{1}\right)=1$.

(2) Applying Lemma 5.1 for $P=\widetilde{D}_{i}, Q=\widetilde{D}-\widetilde{D}_{i}$, we have $p_{a}\left(\widetilde{D}_{i}\right)=$ $-P \cdot Q / 2+1 \leqq 0$. This implies that $\widetilde{D}_{i} \cong P^{1}$. Thus $\left(\widetilde{D}_{i}^{2}-\widetilde{D} \cdot \widetilde{D}_{i}\right) / 2+1=0$. 
(3) Assume that $\widetilde{D}_{i}^{2}>0$. Then $\widetilde{D} \cdot \widetilde{D}_{i}>2$ by (2). Thus $2=\widetilde{H} \cdot \widetilde{D} \geqq \widetilde{H} \cdot \widetilde{D}_{i}=$ $\widetilde{D} \cdot \widetilde{D}_{i}+\widetilde{F} \cdot \widetilde{D}_{i}>2+0=2$. This is a contradiction. Next assme that $\widetilde{D}_{i}^{2}<-4$. Then $\widetilde{D} \cdot \widetilde{D}_{i}<-2$. Thus $2=\widetilde{F} \cdot \widetilde{D} \geqq \widetilde{F} \cdot \widetilde{D}_{i}=\widetilde{H} \cdot \widetilde{D}_{i}-\widetilde{D} \cdot \widetilde{D}_{i}>0+2=2$. This is also a contradiction. Next assume that $\widetilde{D}_{i}^{2}=0$ for some $i$. By the adjunction formula $\omega_{z} \cdot \widetilde{D}_{i}=-2$. Since $H^{2}\left(\mathcal{O}_{Z}\left(\widetilde{D}_{i}\right)\right) \cong H^{0}\left(\mathcal{O}_{z}\left(-\widetilde{D}-\widetilde{D}_{i}\right)\right)=0$, we have by the Riemann-Roch theorem that $H^{0}\left(\mathscr{O}_{Z}\left(\widetilde{D}_{i}\right)\right) \geqq\left(\widetilde{D}_{i}^{2}-\omega_{z} \cdot \widetilde{D}_{i}\right) / 2+$ $\chi\left(\mathscr{O}_{Z}\right)=2$. Thus $H^{0}\left(\mathcal{O}_{Z}(\widetilde{D})\right) \geqq 2$. This contradicts the fact that $\operatorname{dim}|\widetilde{D}|=0$. Therefore $\widetilde{D}_{i}^{2} \neq 0$. Suppose $\widetilde{D}_{i}^{2}=-4$ for some $i$. Setting $D^{\prime}=\widetilde{D}-\widetilde{D}_{i}$, we have $H^{0}\left(\mathcal{O}_{z}\left(D^{\prime}\right)\right) \geqq 2$ by the Riemann-Roch theorem as above. Thus $\widetilde{D}_{i}^{2} \neq$ -4 for every $i$. Therefore $-3 \leqq \widetilde{D}_{i}^{2} \leqq-1$.

(4) We have $\sum(-t+2) N(-t)=0$ since $0=\widetilde{D}^{2}=\sum a_{i} \widetilde{D} \cdot \widetilde{D}_{i}=\sum a_{i}\left(\widetilde{D}_{i}^{2}+2\right)$. Thus $N(-1)=N(-3)$. Moreover we have $0 \leqq N(-1) \leqq \tilde{H} \cdot \widetilde{D}=2$ since if $\widetilde{D}_{i}{ }^{2}=-1$, then $\widetilde{D}_{i}$ is an exceptional curve of the first kind and $\widetilde{D}_{i} \cdot \widetilde{H}>0$ by the minimality of $\rho$.

(5) Note that $0 \leqq \widetilde{H} \cdot \widetilde{D}_{i}=\widetilde{D} \cdot \widetilde{D}_{i}+\widetilde{F} \cdot \widetilde{D}_{i}=\widetilde{D} \cdot \widetilde{D}_{i}=2+\widetilde{D}_{i}^{2}$.

(6) $\widetilde{D}_{i}^{2} \neq-1$ by the minimality of $\rho$.

Q.E.D.

The next proposition is an easy consequence of the above lemma.

Proposition 5.3. We use the same notation as in Lemma 5.2. Assume that $r \geqq 2$. By renumbering if necessary, we can assume that one of the following holds. Note (0) and (2) in Lemma 5.2.

(a) $\widetilde{D}_{i}^{2}=-2$ for every $i$.

(b) $a_{1}=a_{2}=1 ; \widetilde{D}_{1}^{2}=-3, \widetilde{H} \cdot \widetilde{D}_{1}=1 ; \widetilde{D}_{2}^{2}=-1, \widetilde{H} \cdot \widetilde{D}_{2}=1 ;$ and $\widetilde{D}_{j}^{2}=-2, \widetilde{H} \cdot \widetilde{D}_{j}=0$ for every $j \geqq 3$.

(c-1) $\quad a_{1}=a_{2}=1 ; \quad \widetilde{D}_{1}^{2}=-3 ; \quad \widetilde{H} \cdot \widetilde{D}_{1}=0 ; \quad \widetilde{D}_{2}^{2}=-1, \quad \widetilde{H} \cdot \widetilde{D}_{2}=2 ; \quad$ and $\widetilde{D}_{j}^{2}=-2$, $\widetilde{H} \cdot \widetilde{D}_{j}=0$ for every $j \geqq 3$.

(c-2) $\quad r \geqq 3 ; a_{1}=a_{2}=a_{3}=1 ; \widetilde{D}_{1}^{2}=-3, \widetilde{H} \cdot \widetilde{D}_{1}=0 ; \widetilde{D}_{2}^{2}=-1, \widetilde{H} \cdot \widetilde{D}_{2}=1 ; \widetilde{D}_{3}^{2}=-2$, $\widetilde{H} \cdot \widetilde{D}_{3}=1 ;$ and $\widetilde{D}_{j}^{2}=-2$, $\widetilde{H} \cdot \widetilde{D}_{j}=0$ for every $j \geqq 4$.

(d-1) $\quad r \geqq 4 ; a_{1}=a_{2}=a_{3}=a_{4}=1 ; \widetilde{D}_{1}^{2}=\widetilde{D}_{2}^{2}=-3, \widetilde{H} \cdot \widetilde{D}_{1}=\widetilde{H} \cdot \widetilde{D}_{2}=0 ; \widetilde{D}_{3}^{2}=\widetilde{D}_{4}^{2}=-1$, $\widetilde{H} \cdot \widetilde{D}_{3}=\widetilde{H} \cdot \widetilde{D}_{4}=1 ;$ and $\widetilde{D}_{j}^{2}=-2, \widetilde{H} \cdot \widetilde{D}_{j}=0$ for every $j \geqq 5$.

$(\mathrm{d}-2) \quad r \geqq 3 ; \quad a_{1}=a_{2}=1, \quad a_{3}=2 ; \quad \widetilde{D}_{1}^{2}=\widetilde{D}_{2}^{2}=-3, \quad \widetilde{H} \cdot \widetilde{D}_{1}=\widetilde{H} \cdot \widetilde{D}_{2}=0 ; \quad \widetilde{D}_{3}^{2}=-1$, $\widetilde{H} \cdot \widetilde{D}_{3}=1 ; \widetilde{D}_{j}^{2}=-2, \tilde{H} \cdot \widetilde{D}_{j}=0$ for every $j \geqq 4$.

(d-3) $\quad r \geqq 3 ; \quad a_{1}=2, \quad a_{2}=a_{3}=1 ; \quad \widetilde{D}_{1}^{2}=-3, \quad \widetilde{H} \cdot \widetilde{D}_{1}=0 ; \quad \widetilde{D}_{2}^{2}=\widetilde{D}_{3}^{2}=-1, \quad \widetilde{H} \cdot \widetilde{D}_{2}=$ $\widetilde{H} \cdot \widetilde{D}_{3}=1 ;$ and $\widetilde{D}_{j}^{2}=-2, \widetilde{H} \cdot \widetilde{D}_{j}=0$ for every $j \geqq 4$.

(d-4) $\quad a_{1}=a_{2}=2 ; \quad \widetilde{D}_{1}^{2}=-3, \quad \tilde{H} \cdot \widetilde{D}_{1}=0 ; \quad \widetilde{D}_{2}^{2}=-1, \quad \tilde{H} \cdot \widetilde{D}_{2}=1 ; \quad$ and $\widetilde{D}_{j}^{2}=-2$, $\widetilde{H} \cdot \widetilde{D}_{j}=0$ for every $j \geqq 3$.

Proof. First assume that $N=0$. We have Case (a).

Secondly assume that $N=1$. We can assume that $\widetilde{D}_{1}^{2}=-3, \widetilde{D}_{2}^{2}=-1$, $a_{1}=a_{2}=1$ and $\widetilde{D}_{j}^{2}=-2$ for $j \geqq 3$ by Lemma $5.2(4)$. By Lemma $5.2(6)$ 
$2=\widetilde{H} \cdot \widetilde{D} \geqq \widetilde{H} \cdot \widetilde{D}_{2}>0$. If $\widetilde{H} \cdot \widetilde{D}_{2}=2$, then $\widetilde{H} \cdot \widetilde{D}_{j}=0$ for $j \neq 2$ and we have Case (c-1). If $\tilde{H} \cdot \widetilde{D}_{2}=1$, then we have Case (b) or (c-2) according as $\widetilde{H} \cdot \widetilde{D}_{1}=1$ or 0 .

Thirdly assume that $N=2$. We can assume that $\widetilde{D}_{1}^{2}=-3$. We have $2=N \geqq a_{1}>0$. If $a_{1}=1$, we can assume moreover that $\widetilde{D}_{2}^{2}=-3, a_{2}=1$ and $\widetilde{D}_{3}^{2}=-1$ by (4). In this case $2=\widetilde{H} \cdot \widetilde{D} \geqq a_{3} \widetilde{H} \cdot \widetilde{D}_{3}>0$ by (6). If $a_{3}=2$, we have that $\widetilde{H} \cdot \widetilde{D}_{3}=1$ and that $\widetilde{H} \cdot \widetilde{D}_{j}=0$ for $j \neq 3$. Lemma 5.2 (4) implies that $\widetilde{D}_{j}^{2}=-2$ for $j \geqq 4$. We have Case (d-2). If $a_{3}=1$, then we can assume that $\widetilde{D}_{4}^{2}=-1$. By (6) $\widetilde{H} \cdot \widetilde{D}_{3}>0$ and $\widetilde{H} \cdot \widetilde{D}_{4}>0$. We have that $\widetilde{H} \cdot \widetilde{D}_{j}=0$ for $j \neq 3,4$ and that $\widetilde{H} \cdot \widetilde{D}_{3}=\widetilde{H} \cdot \widetilde{D}_{4}=1$ since $\tilde{H} \cdot\left(\sum a_{j} \widetilde{D}_{j}\right)=2$. By (4) $\widetilde{D}_{j}^{2}=-2$ for $j \geqq 5$. We have Case (d-1).

If $2=N=a_{1}$, then we can assume that $\widetilde{D}_{2}^{2}=-1$. Note that $2=\widetilde{H} \cdot \widetilde{D} \geqq$ $a_{2} \tilde{H} \cdot \widetilde{D}_{2}>0$. If $a_{2}=2$, then $\widetilde{H} \cdot \widetilde{D}_{2}=1$ and $\widetilde{H} \cdot \widetilde{D}_{j}=0$ for $j \neq 2$. In this case $\widetilde{D}_{j}^{2}=-2$ for $j \geqq 3$ by (4). We have Case (d-4). If $a_{2}=1$, we can assme that $\widetilde{D}_{3}^{2}=-1$ and similarly we have Case (d-3).

Now by the above argument they are all posibilities.

In the following by $\bar{B}$ (resp. $\bar{C}$ ) we denote the section of $\tau: \Sigma_{2} \rightarrow P^{1}$ with $\bar{B}^{2}=-2$ (resp. $\left.\bar{C}^{2}=+2\right) . \quad \bar{F}$ is a general fibre of $\tau$.

LEMMA 5.4. Let

$$
\sigma: Z=Z_{8} \stackrel{\sigma_{8}}{\longrightarrow} Z_{7} \stackrel{\sigma_{7}}{\longrightarrow} Z_{8} \longrightarrow \cdots \longrightarrow Z_{1} \stackrel{\sigma_{1}}{\longrightarrow} Z_{0}=\Sigma_{2}
$$

be an admissible blowing-down to $\Sigma_{2}$. Let $z_{i} \in Z_{i-1}$ be the center of $\sigma_{i}$. Assume that either the following (A) or (B) is satisfied.

(A) The image of $z_{i}$ on $Z_{0}=\Sigma_{2}$ does not belong to $\bar{B}$ for some $i$ with $1 \leqq i \leqq 8$.

(B) For some point $x \in \bar{B}$ there is a smooth curve $\bar{K} \subset \Sigma_{2}$ passing through $x$ such that $\bar{K}$ is tangent to neither $\bar{B}$ nor the fibre of $\tau$ passing through $x$ and such that for some three indices $i, j, k$ with $1 \leqq i<j<k \leqq 8, z_{i}, z_{j}$ and $z_{k}$ coincide with the intersection point lying over $x$ of the strict transform of $\bar{K}$ and the total transform of $\bar{B}$.

Then there is another admissible blowing-down $\hat{\sigma}: Z \rightarrow P^{2}$ to $P^{2}$.

Proof. First assume that (A) is satisfied. By exchanging the order of blowing-ups, we can assume that $z_{1} \notin \bar{B}$. Let $F^{\prime} \subset Z_{1}$ be the strict transform on $Z_{1}$ of the fibre of $\tau$ passing through $z_{1} . \quad F^{\prime}$ is an exceptional curve of the first kind. The contraction of $F^{\prime}$ defines a morphism $Z_{1} \rightarrow \Sigma_{1}$. We have an admissible blowing-down $Z_{2}^{\sigma_{2} \sigma_{3} \cdots \sigma_{8}} \longrightarrow Z_{1} \rightarrow \Sigma_{1} \rightarrow P^{2}$.

Next assume that (B) is satisfied. By exchanging the order of blowingups we can assume that $i=1, j=2$ and $k=3$. Consider $Z_{3}$. Let $\hat{E}_{\alpha}$ be 
the strict transform of $\sigma_{\alpha}^{-1}\left(z_{\alpha}\right)$ on $Z_{3}$ for $1 \leqq \sigma \leqq 3$. Let $\hat{F}$ (resp. $\hat{K}$ ) be the strict transform on $Z_{3}$ of the fibre of $\tau$ passing through $x=z_{1}$ (resp. of $\bar{K}$ ). Their configuration is represented by the next dual graph. (The attached integer is the self intersection number. The intersection of $\hat{K}$ and $\hat{F}$ and that of $\hat{K}$ and $\hat{B}$ are omitted.)

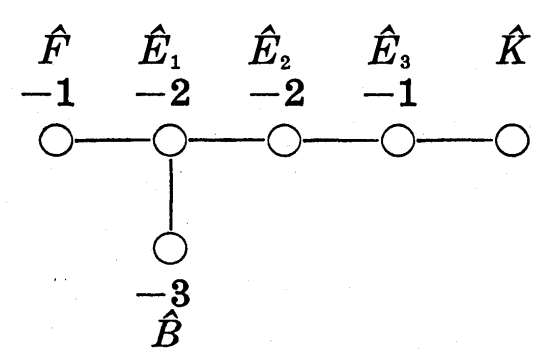

We can contract successively $\hat{F}, \hat{E}_{1}, \hat{E}_{2}, \hat{B}$ in order. We can check easily that this defines an admissible blowing-down $Z=Z_{8} \rightarrow Z_{3} \rightarrow \Sigma_{1} \rightarrow P^{2}$. Q.E.D.

LEMMA 5.5. Let $\bar{D} \in\left|-\omega_{\Sigma_{2}}\right|$ be an effective anti-canonical divisor on $\Sigma_{2}$. Assume that Supp $\bar{D} \cap \bar{B} \neq \varnothing$. Then one of the following holds.

(1) $\bar{D}=\bar{B}+\bar{G}, \bar{G}$ is a section of $\tau$ with $\bar{G}^{2}=6$ and $\bar{B}$ and $\bar{G}$ intersect at 2 points transversally.

(2) $\bar{D}=\bar{B}+\bar{G}$ and $\bar{G}$ is the same as in (1). However, $\bar{B}$ and $\bar{G}$ are tangent at one point.

(3) $\bar{D}=\bar{B}+\bar{G}+\bar{F}_{1}, \bar{G}$ is a section of $\tau$ with $\bar{G}^{2}=4$ and $\bar{F}_{1}$ is a fibre of $\tau$.

(4) $\bar{D}=\bar{B}+\bar{C}+\bar{F}_{1}+\bar{F}_{2}, \bar{C}$ is a section of $\tau$ with $\bar{C}^{2}=2$ and $\bar{F}_{1}$ and $\bar{F}_{2}$ are fibres of $\tau$.

(5) $\bar{D}=2 \bar{B}+\bar{F}_{1}+\bar{F}_{2}+\bar{F}_{3}+\bar{F}_{4}$ and $\bar{F}_{i}$ is a fibre of $\tau$ for $1 \leqq i \leqq 4$.

Proof. Assume that $\bar{D}$ does not contain $\bar{B}$. Then $\bar{D} \cdot \bar{B}>0$ since Supp $\bar{D} \cap \bar{B} \neq \varnothing$. On the other hand by the adjunction formula we have $\bar{D} \cdot \bar{B}=0$, a contradiction. Thus $\bar{D}$ contains $\bar{B}$.

Since $\bar{F} \cdot(\bar{D}-\bar{B})=2-1=1, \bar{D}$ contains another section $\bar{G}$ and $\bar{D}=$ $\bar{B}+\bar{G}+\bar{F}_{1}+\cdots+\bar{F}_{a}$ for some fibres $\bar{F}_{i}$ of $\tau$. For some integer $b, \bar{G} \sim$ $\bar{B}+b \bar{F}$. We have $b=(B+b \bar{F}) \cdot \bar{C}=\bar{G} \cdot \bar{C} \geqq 0$. Moreover since $2 \bar{B}+4 \bar{F} \sim 2 \bar{B}+$ $(a+b) \bar{F}, a+b=4$ and $b \leqq 4$.

If $b=0$, then $\bar{G}=\bar{B}$ and we have Case (5).

Assume $b>0$. Then $\bar{G} \neq \bar{B}$ and we have $-2+b=(\bar{B}+b \bar{F}) \cdot \bar{B}=\bar{G} \cdot \bar{B} \geqq 0$. If $b=2$, we have Case (4). If $b=3$, Case (3) takes place. When $b=4$, we have Case (1) or (2) according as $\bar{B}$ and $\bar{G}$ are tangent or not. Q.E.D.

Proposition 5.6. $Z$ has an admissible blowing-down to $\boldsymbol{P}^{2}$. 
Proof. By Proposition 4.5 $Z$ has an admissible blowing-down to $\boldsymbol{P}^{2}$ or $\Sigma_{2}$. Let $\sigma: Z \rightarrow \Sigma_{2}$ be an admissible blowing-down to $\Sigma_{2}$ We use the notation in Lemma 5.4. We have only to show that if Condition (A) in Lemma 5.4 is not satisfied, then (B) in the same lemma holds. Thus in the following we assume that all $z_{1}, z_{2}, \cdots, z_{8}$ lie on the total transform of $\bar{B}$.

By Lemma $2.4 z_{1} \in \bar{B} \cap \operatorname{Supp} \bar{D}$ for $\bar{D}=\sigma_{*} \widetilde{D} \in\left|-\omega_{\Sigma_{2}}\right|$. Thus one of (1)(5) in Lemma 5.5 holds.

Assume that (1) holds. We will show that (B) holds for $\bar{K}=\bar{G}$. Note that the strict transform of $\widetilde{G}$ on $Z$ of $\bar{G}$ satisfies $\widetilde{G}^{2} \leqq-1$ by Lemma 5.2 (3). Thus at least $7=\bar{G}^{2}-(-1)$ of $z_{1}, \cdots, z_{8}$ lie on the strict transform of $\bar{G}$. The images of these 7 points belong to $\bar{B} \cap \bar{G}$ under our assumption. Thus for one point $x$ of the two points in $\bar{B} \cap \bar{G}$, (B) is satisfied.

Assume that (2) holds. Let $G_{1}$ denote the strict transform of $\bar{G}$ on $Z_{1}$. At least 6 of $z_{2}, \cdots, z_{8}$ lie on the strict transform of $G_{1}$. By exchanging the order of blowing-ups, we can assume that $z_{2} \in G_{1}$. Let $B_{1}$ denote the strict transform of $\bar{B}$ on $Z_{1}$. Note that in this case the intersection of $G_{1}$ and the total transform of $\bar{B}$ coincides with $G_{1} \cap B_{1}$. Thus $z_{2} \in B_{1}$. Since $B_{1}^{2}=-3$, the strict transform $\widetilde{B}$ of $\bar{B}$ on $Z$ satisfies $\widetilde{B}^{2} \leqq-4$, which contradicts Lemma 5.2 (3).

Assume that (3) holds. In this case $\bar{B}$ and $\bar{G}$ intersect transversally at one point $x$. We will show that (B) holds for $\bar{K}=\bar{G}$. By Lemma 5.2 (3) the strict transform $\widetilde{G}$ of $\bar{G}$ on $Z$ satisfies $\widetilde{G}^{2} \leqq-1$. Thus at least $5=\bar{G}^{2}-(-1)$ points of $z_{1}, \cdots, z_{8}$ lie on the strict transform of $\bar{G}$. Under our assumption these 5 points lie over $x$. Thus (B) is satisfied.

Assume that (4) holds. In this case $\bar{B} \cap \bar{C}=\varnothing$. Thus none of $z_{1}, \cdots, z_{8}$ lies on the strict transform of $\bar{C}$. Thus the strict transform $\widetilde{C}$ of $\bar{C}$ on $Z$ satisfies $\widetilde{C}^{2}=\bar{C}^{2}=2$, which contradicts Lemma $5.2(3)$.

Lastly assume that (5) holds. By Lemma $4.4 z_{i}$ lies on the strict transform of $\bar{H}=\sigma_{*} \widetilde{H}$ for $1 \leqq i \leqq 8$ and $\bar{H} \sim 2 \bar{C}+\bar{F}$. The intersection $\bar{H} \cap \bar{B}$ is one point $x$ and $\bar{H}$ and $\bar{B}$ are transversal since $\bar{H} \cdot \bar{B}=1$. Let $\bar{F}_{0}$ be the fibre of $\tau$ passing through $x$. If $\bar{H}$ is not tangent to $\bar{F}_{0}$ at $x$, then (B) is satisfied for $\bar{K}=\bar{H}$. Thus in the following we consider the case where $\bar{H}$ is tangent to $\bar{F}_{0}$ at $x$ and $\bar{D}=2 \bar{B}+\bar{F}_{1}+\bar{F}_{2}+\bar{F}_{3}+\bar{F}_{4}$, where $\bar{F}_{i}$ is a fibre of $\tau$.

Under our assumption the image of $z_{i}$ on $Z_{0}=\Sigma_{2}$ coincides with $x$ for $1 \leqq i \leqq 8$. If $\bar{F}_{i}$ does not pass through $x$ for some $i$ with $1 \leqq i \leqq 4$, then the strict transform $\widetilde{F}_{i}$ of $\bar{F}_{i}$ on $Z$ has self intersection number $\widetilde{F}_{i}^{2}=\bar{F}_{i}^{2}=0$, which contradicts Lemma 5.2 (3). Thus $\bar{F}_{0}=\bar{F}_{1}=\bar{F}_{2}=\bar{F}_{8}=\bar{F}_{4}$.

Let $\widetilde{F}$ (resp. $\widetilde{B}$ ) be the strict transform of $\bar{F}_{0}$ (resp. $\bar{B}$ ) on $Z$. Let $\widetilde{E}_{i}$ denote the strict transform of $\sigma_{i}^{-1}\left(z_{i}\right)$ on $Z$. By Lemma 4.4 (1) and 
by the fact $\bar{F} \cdot \bar{H}=2$ one sees that their configuration is represented by the next dual graph. (The attached integer is the self intersection number.)

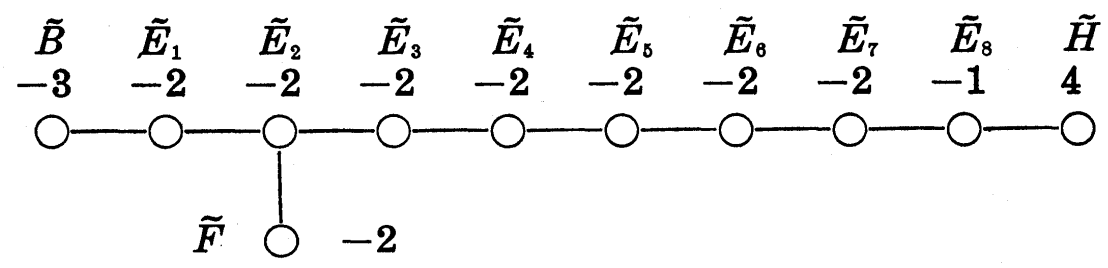

It can be seen that $\widetilde{D}=2 \widetilde{B}+5 \widetilde{E}_{1}+8 \widetilde{E}_{2}+7 \widetilde{E}_{3}+6 \widetilde{E}_{4}+5 \widetilde{E}_{5}+4 \widetilde{E}_{6}+3 \widetilde{E}_{7}+2 \widetilde{E}_{8}+4 \widetilde{F}$. Set $D^{\prime}=\widetilde{E}_{1}+2 \sum_{i=2}^{8} \widetilde{E}_{i}+\widetilde{F}$. $\widetilde{D}-D^{\prime}$ is effective and it is easy to see that $D^{\prime}=\sigma^{*} \bar{F}_{0} . \quad$ Thus $\operatorname{dim}|\widetilde{D}| \geqq \operatorname{dim}\left|D^{\prime}\right|=\operatorname{dim} \sigma^{*}\left|\bar{F}_{0}\right| \geqq 1$, which contradicts Proposition 4.6.

Q.E.D.

REMARK. The essential part of the above proposition has been obtained by Clebsch in [5]. He studied a quartic surface in $\boldsymbol{P}^{3}$ whose onedimensional singular locus is a line. He showed that if it contains certain quadratic curves, then it is the image of a rational mapping corresponding to the linear system of quartic curves on $P^{2}$ with 9 base points and he studied properties of this rational mapping. He gave the classification of lines and quadratic curves on the surface, too.

Moreover he studied in [5] a quintic surface whose one-dimensional singular locus is either a cubic curve or two lines in twisted position. He studied it as the image of $\boldsymbol{P}^{2}$ by a rational mapping.

Now in order to complete the proof of our main results in section 1 we need further several lemmas and propositions.

LEMma 5.7. Let $A$ be a reduced irreducible curve on $Z$ such that $\tilde{H} \cdot A=0$. Then one of the following holds.

(1) $A$ is a smooth rational curve, $A \subset \operatorname{Supp} \widetilde{D}$, and $A^{2}=-2$ or -3 .

(2) $A$ is a smooth rational curve, $A \cap \operatorname{Supp} \widetilde{D}=\varnothing$ and $A^{2}=-2$.

Proof. If $A \subset \operatorname{Supp} \widetilde{D}$, by Lemma 5.2 we obtain the conclusion. Assume that $A \not \subset \operatorname{Supp} \widetilde{D}$. Thus $A \cdot \widetilde{D} \geqq 0$. By the Hodge index theorem $A^{2}<0$. Since $A^{2}-A \cdot \widetilde{D}=2 p_{a}(A)-2 \geqq-2$, either " $A^{2}=-2$ and $A \cdot \widetilde{D}=0$ " or " $A^{2}=-1$ and $A \cdot \widetilde{D}=1$ " holds. If the latter case takes place, then $0 \leqq$ $\widetilde{F} \cdot A=\widetilde{H} \cdot A-\widetilde{D} \cdot A=-1$, which is a contradiction. Note that the condition $A \cdot \widetilde{D}=0$ implies that $A \cap \operatorname{Supp} \widetilde{D}=\varnothing$.

Q.E.D.

LEMMA 5.8. Set $I=\left\{i \mid 1 \leqq i \leqq r, \widetilde{D}_{i} \cdot \widetilde{H}=0\right\}$ and $D^{\prime}=\sum_{i \in I} a_{i} \widetilde{D}_{i}$. For any connected component $D^{(0)}$ of $D^{\prime}$, Supp $D^{(0)}$ is the union of exceptional curves of the minimal resolution of a rational normal singular point. 
Proof. Let $D_{1}^{(0)}, \cdots, D_{t}^{(0)}$ be all irreducible components of $D^{(0)}$. $\tilde{H} \cdot D_{i}^{(0)}=$ 0 for $1 \leqq i \leqq t$. By the Hodge index theorem the matrix $\left(D_{i}^{(0)} \cdot D_{j}^{(0)}\right)_{1 \leq t, j \leq t}$ is negative definite. Furthermore any $D_{i}^{(0)}$ is not an exceptional curve of the first kind by Lemma 5.2. Therefore by the Grauert's theorem Supp $D^{(0)}$ is the union of exceptional curves of the minimal resolution of some normal singular point. Next we show that this normal singular point is rational. Set $D^{(1)}=\widetilde{D}-D^{(0)}$. We have $H^{2}\left(\mathcal{O}_{z}\left(-D^{(0)}\right)\right) \cong H^{0}\left(\mathcal{O}_{z}\left(-D^{(1)}\right)\right)=0$, by the Serre duality. By the exact sequence $0 \rightarrow \mathscr{O}_{Z}\left(-D^{(0)}\right) \rightarrow \mathcal{O}_{Z} \rightarrow \mathcal{O}_{D^{(0)} \rightarrow 0 \text {, }}$ one can conclude that $H^{1}\left(\mathscr{O}_{D^{(0)}}\right)=0$. Now on the other hand note that $\left(-D^{(0)}-\omega_{z}\right) \cdot D_{i}^{(0)} \geqq 0$ for $1 \leqq i \leqq t$, since $-D^{(0)}-\omega_{z} \sim D^{(1)}$ and since $D^{(0)}$ and $D^{(1)}$ have no common components. By a vanishing theorem due to Kato (Kato [13]) under this condition one has $H^{1}\left(\mathcal{O}_{U}\left(-D^{(0)}\right)\right)=0$ where $U$ is a sufficiently small neighbourhood of Supp $D^{(0)}$. By the exact sequence

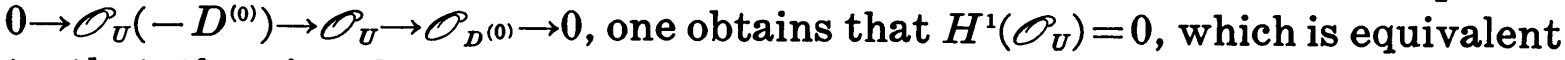
to that the singular point is rational.

Q.E.D.

By the above two lemmas, the Hodge index theorem and the characterization of rational double point due to Artin we have the following.

Proposition 5.9. Let $E \subset Z$ be the union of irreducible curves $A$ on $Z$ with $\tilde{H} \cdot A=0$. Let $E_{0}$ be an arbitrarily chosen connected component of $E$. Then one of the following (1), (2) necessarily holds.

(1) $E_{0}$ is a connected component of the support of the divisor which is obtained from $\widetilde{D}=\sum a_{i} \widetilde{D}_{i}$ by removing all the components $\widetilde{D}_{j}$ with $\widetilde{D}_{j} \cdot \widetilde{H}>0 . \quad E_{0}$ coincides with the union of exceptional curves of the minimal resolution of some normal isolated rational singular point.

(2) $E_{0} \cap \operatorname{Supp} \widetilde{D}=\varnothing$ and $E_{0}$ coincides with the union of exceptional curves of the minimal resolution of a rational double point.

Corollary 5.10. (1) If $\widetilde{D}$ has the form (a) or (b) in Proposition 5.3 , then all singular points on the normalization $\hat{X}$ are rational double points.

(2) If $\widetilde{D}$ has form (c) or (d) in Proposition 5.3, then $\hat{X}$ has rational triple points on the inverse image $\hat{D}$ of the one-dimensional singular locus. The number of rational triple points on $\hat{X}$ is at most 2 . All other singular points on $\hat{X}$ than rational triple points on $\hat{D}$ are rational double points. The images of these rational triple points by the morphism $\nu: \hat{X} \rightarrow X$ are also triple points.

Proof. (1) By Artin [2] it is obvious. (2) The image on $\hat{X}$ of the component $\widetilde{D}_{j}$ of $\widetilde{D}$ with $\widetilde{D}_{j}^{2}=-3, \widetilde{D}_{j} \cdot \widetilde{H}=0$ is not a rational double point but it is a rational singular point. If its multiplicity is greater than 3 , 
then it is 4 since $X$ has degree 4 and $X$ is a cone over a plane quartic curve. If $X$ has a point with multiplicity 4, obviously the minimal resolution $Z$ of the normalization $\hat{X}$ is $\Sigma_{4}$. In our case $Z$ is not $\Sigma_{4}$. Thus its multiplicity is 3 and its image on $X$ is also a triple point. Since the number of $\widetilde{D}_{j}$ with $\widetilde{D}_{j}^{2}=-3$ is at most 2 by Proposition 5.3, the number of triple points on $\hat{X}$ is at most 2 .

Q.E.D.

REMARK. In [21] Rohn gave a precise description of quartic surfaces with a triple point. In particular he knew that there is a quartic surface in $\boldsymbol{P}^{3}$ whose one-dimensional singular locus is a line and such that it has one or two triple points on the singular line.

\section{§6. Special blowing-downs.}

This section is the continuation of Section 5 . We deal with the same surface $Z$ as in Section 5 . We show that there is a special blowing-down in case (b), (c) and (d) in Proposition 5.3.

Now we have an admissible blowing-down to $\boldsymbol{P}^{2}$ by Proposition 5.6.

$$
\sigma: Z=Z_{9} \stackrel{\sigma_{9}}{\longrightarrow} Z_{8} \stackrel{\sigma_{8}}{\longrightarrow} Z_{7} \longrightarrow \cdots \longrightarrow Z_{1}=\Sigma_{1} \stackrel{\sigma_{1}}{\longrightarrow} Z_{0}=\boldsymbol{P}^{2}
$$

Let $z_{i} \in Z_{i-1}$ be the center of $\sigma_{i}$. We define inductively $D_{9}=\widetilde{D}, H_{9}=\tilde{H}$, $D_{i-1}=\sigma_{i *} D_{i}$ and $H_{i-1}=\sigma_{i *} H_{i}$ for $k \geqq i \geqq 1$. By Proposition $4.5 \bar{H}=H_{0}$ is a reduced irreducible quartic curve with only one singular point of type $A_{1}$ or $A_{2}$ at $z_{1}$.

Consider the next two conditions in particular.

(a) The line in $\boldsymbol{P}^{2}$ passing through $z_{1}$ and $z_{2}$ does not pass through $z_{3}$.

(b) The strict transform of the exceptional curve $\sigma_{1}^{-1}\left(z_{1}\right)$ does not pass through both $z_{2}$ and $z_{3}$.

We can check that under (a) and (b) the linear system $\left|2 \hat{E}_{0}-\hat{E}_{1}-\hat{E}_{2}-\hat{E}_{3}\right|$ on $Z_{3}\left(\hat{E}_{0}\right.$ is the total transform on $Z_{3}$ of a line in $P^{3} . \hat{E}_{i}$ is the total transform of the $i$-th exceptional curve $\sigma_{i}^{-1}\left(z_{i}\right)$ for $i \geqq 1$.) defines a birational morphism $\gamma: Z_{3} \rightarrow P^{2}$. The composition $\gamma\left(\sigma_{1} \sigma_{2} \sigma_{3}\right)^{-1}: P^{2}-\cdots \rightarrow P^{2}$ is the birational mapping called the quadratic transformation with center $z_{1}, z_{2}$ and $z_{3}$. We can check that $\gamma=\gamma_{1} \gamma_{2} \gamma_{3}$ is a composition of three blowingups $\gamma_{1}, \gamma_{2}, \gamma_{3}$ such that $\tau \sigma_{2} \sigma_{3}=\tau \gamma_{2} \gamma_{3}$ for the projection $\tau: \Sigma_{1} \rightarrow P^{1}$. Thus $\hat{\sigma}=\gamma \sigma_{4} \sigma_{5} \cdots \sigma_{9}$ defines another admissible blowing-down to $\boldsymbol{P}^{2}$.

Proposition 6.1. Assume that $\widetilde{D}$ contains a component $\widetilde{D}_{j}$ with $\widetilde{D}_{j}^{2}=-3$ and $\widetilde{D}_{j} \cdot \widetilde{H}=0$. Then there is an admissible blowing-down $\sigma: Z \rightarrow \boldsymbol{P}^{2}$ to $\boldsymbol{P}^{2}$ with $\sigma^{*} \widetilde{D}_{j}=0$. 
Proof. By exchanging indices if necessary, we assume that $\widetilde{D}_{1}^{2}=-3$ and $\widetilde{D}_{1} \cdot \widetilde{H}=0$. By Proposition 5.6 there is an admissible blowing-down $\sigma$ to $\boldsymbol{P}^{2}$. We use the above notation. We will show that by modifying the above sequence $\left(^{*}\right)$, we can obtain the desired blowing-down. Note that $1 \leqq$ degree of $\sigma_{*} \widetilde{D}_{1} \leqq 3$, if $\sigma_{*} \widetilde{D}_{1} \neq 0$, since $D_{0}=\sigma_{*} \widetilde{D} \in\left|-\omega_{P 2}\right|=$ the linear system of cubic curves.

Case (1). $\quad \sigma_{*} \widetilde{D}_{1}$ is a cubic curve in $P^{2}$.

If $A=\sigma_{*} \widetilde{D}_{1}$ is smooth, then we have $\widetilde{D}_{1}^{2}=\left(\sigma_{*} \widetilde{D}_{1}\right)^{2}-9=0$, a contradiction. Thus $A$ is a reduced irreducible singular cubic curve. Let $z$ be its unique singular point. The singularity $(A, z)$ is of type $A_{1}$ or $A_{2}$. Assume for the moment that $z \neq z_{1}$. The strict transform $\left(\sigma_{2} \sigma_{3} \cdots \sigma_{\theta}\right)_{*} \widetilde{D}_{1}$ of $A$ by $\sigma_{1}$ intersects with the strict transform $\left(\sigma_{2} \cdots \sigma_{\vartheta}\right)_{*} \widetilde{F}$ of $\bar{F}$ by $\sigma_{1}$ at two points. If $\widetilde{F}$ is a sufficiently general member of $\left|\widetilde{E}_{0}-\widetilde{E}_{1}\right|$, this implies that $\widetilde{D}_{1} \cdot \widetilde{F}=2$. On the other hand $\widetilde{D} \cdot \widetilde{D}_{1}=-1$ since $\widetilde{D}_{1}^{2}=-3$ and $p_{a}\left(\widetilde{D}_{1}\right)=0$. Therefore $\widetilde{D}_{1} \cdot \widetilde{H}=\widetilde{D}_{1} \cdot \widetilde{F}+\widetilde{D}_{1} \cdot \widetilde{D}=1 \neq 0$, which contradicts the assumption. One sees that $z=z_{1}$. Now since $\widetilde{D}_{1}^{2}=-3$, all of $z_{1}, z_{2}, \cdots, z_{9}$ lie on the strict transform of $A$. The line passing through $z_{1}$ and $z_{2}$ does not pass through $z_{3}$ since $z_{1}$ is a double point of $A$. The above Condition (a) is satisfied.

Subcase (1-1). The image of $z_{i}$ on $Z_{0}$ is different from $z=z_{1}$ for some $i$ with $2 \leqq i \leqq 9$.

By exchanging the order of blowing-ups, we can assume that $z_{1} \neq \sigma_{1}\left(z_{2}\right)$. Then the above Condition (b) is also satisfied and we can define another admissible blowing-down $\hat{\sigma}=\gamma \sigma_{4} \sigma_{5} \cdots \sigma_{9}$. By easy calculation one sees that $\hat{\sigma}_{*} \widetilde{D}_{1}$ is a quadratic curve. Thus Subcase (1-1) is reduced to the following Case (2).

Subcase (1-2). The image of $z_{i}$ coincides with the point $z$ for every $i$ with $1 \leqq i \leqq 9$ and $A=\sigma_{*} \widetilde{D}_{1}$ has an $A_{1}$-singularity at $z$.

Let $A^{\prime}=\left(\sigma_{2} \sigma_{3} \cdots \sigma_{9}\right)_{*} \widetilde{D}_{1}$ be the strict transform of $A$ by $\sigma_{1} \cdot A^{\prime}$ intersects with $E^{\prime}=\sigma_{1}^{-1}\left(z_{1}\right)$ at two points $x, y$ transversally. For every $i$ with $2 \leqq i \leqq 9, z_{i}$ lies on the strict transform of $A^{\prime}$ and it lies also on the total transform of $E^{\prime}$ by assumption. Thus for $2 \leqq i \leqq 9$, the image of $z_{i}$ on $Z_{1}$ coincides with either $x$ or $y$. It implies that for either $x$ or $y$, there is two indices $\alpha, \beta$ with $2 \leqq \alpha<\beta \leqq 9$ such that the images of $z_{\alpha}$ and $z_{\beta}$ coincides with it. By exchanging the order of blowing-ups we can assume $\alpha=2, \beta=3$ and $z_{2}=\sigma_{3}\left(z_{3}\right) \in A^{\prime} \cap E^{\prime}$. Note that since $z_{3}$ lies on the strict transform of $A^{\prime}$, it does not lie on the strict transform of $E^{\prime}$. Thus the above Condition (b) is also satisfied and Subcase (1-2) is also reduced to Case (2).

Subcase (1-3). The image of $z_{i}$ coincides with the point $z$ for every $i$ with $1 \leqq i \leqq 9$ and $A=\sigma_{*} \widetilde{D}_{1}$ has an $A_{2}$-singularity at $z$. 
Note that the point $z_{i} \in Z_{i-1}$ is uniquely determined for every $i$ with $1 \leqq i \leqq 9$ by the above condition. In this case both $z_{2}$ and $z_{3}$ lie on the strict transform of $\sigma_{1}^{-1}\left(z_{1}\right)$ and we cannot apply the quadratic transformation.

Instead of it, consider $Z_{5}$. Let $\hat{E}_{i}$ be the strict transform of $\sigma_{i}^{-1}\left(z_{i}\right)$ on $Z_{5}$ for $1 \leqq i \leqq 5$. Let $\hat{F}$ be the strict transform on $Z_{5}$ of the tangent line to $A$ at $z$ (The line which has the local intersection number 3 with $A$ at $z)$ and $\hat{A}=\left(\sigma_{8} \sigma_{7} \sigma_{8} \sigma_{9}\right)_{*} \widetilde{D}_{1}$ be the strict transform of $A$ on $Z_{5}$. Their configuration is represented by the next dual graph. (The attached integer is the self intersection number.)

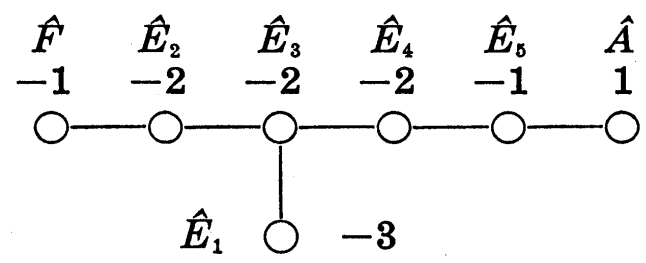

We can contract successively $\widehat{F}, \widehat{E}_{2}, \widehat{E}_{3}, \widehat{E}_{4}, \widehat{E}_{1}$ in order. We have another admissible blowing-down $\sigma^{\prime}: Z \stackrel{\sigma_{8} \sigma_{7} \sigma_{\sigma} \sigma_{9}}{\longrightarrow} Z_{5} \rightarrow \Sigma_{1} \rightarrow P^{2}$. We can check that $\sigma^{\prime}{ }_{*} \widetilde{D}_{1}$ is a line. Thus Subcase (1-3) is reduced to the following Case (3). Case (2). $\quad \sigma_{*} \widetilde{D}_{1}$ is a quadratic curve in $\boldsymbol{P}^{2}$.

Just seven of $z_{1}, z_{2}, \cdots, z_{9}$ lie on the strict transform of $A=\sigma_{*}\left(\widetilde{D}_{1}\right)$ since $A^{2}-\widetilde{D}_{1}^{2}=7$. If $z_{1} \notin A$, then we have $\widetilde{F} \cdot \widetilde{D}=2$, a contradiction. By exchanging the order of blowing-ups we can assume that $z_{1}, z_{2}, \cdots, z_{7}$ lie on the strict transform of $A$. Three points $z_{1}, z_{2}, z_{3}$ do not lie on the same line since they lie on a smooth quadratic curve $A$. Note that the strict transform $A^{\prime}$ of $A$ on $Z_{1}$ intersects with $E^{\prime}=\sigma_{1}^{-1}\left(z_{1}\right)$ at one point transversally since $A$ is smooth. Thus if $z_{2} \in E^{\prime}$, then the strict transform $E^{\prime \prime}$ of $E^{\prime}$ on $Z_{2}$ is disjoint from that of $A$ and $z_{3} \notin E^{\prime \prime}$. One sees that the above Condition (a) and (b) are satisfied. We have another admissible blowing-down $\hat{\sigma}=\gamma \sigma_{4} \cdots \sigma_{\theta}$. Then by calculation one sees that $\hat{\sigma}_{*} \widetilde{D}_{1}$ is a line. Thus Case (2) is reduced to Case (3).

Case (3). $\quad \sigma_{*} \widetilde{D}_{1}$ is a line in $\boldsymbol{P}^{2}$.

Just four of $z_{1}, z_{2}, \cdots, z_{\ominus}$ lie on the strict transform of $A=\sigma_{*} \widetilde{D}_{1}$. If $z_{1} \in A$, then on $Z_{1}$ the intersection number of $H_{1}$ and the strict transform of $A$ is 2. Since $z_{1}, \cdots, z_{8}$ lie on the strict transform of $H_{1}$, at most two of them can lie on the strict transform of $A$. Thus we cannot blow up four times on $A$. One sees that $z_{1} \notin A$. Now by exchanging the order of blowing-ups we can assume that $z_{2}, z_{3}, z_{4}, z_{5}$ lie on the strict transform of $A$. Then we can define the quadratic transformation with center $z_{1}, z_{2}$ and $z_{3}$ and we have another admissible blowing-down $\hat{\sigma}$. For this $\hat{\sigma}$, one 
has $\hat{\sigma}_{*} \widetilde{D}_{1}=0$.

Q.E.D.

Remark. For the blowing-down $\sigma$ in Proposition 5.7, $\widetilde{D}_{j}$ is the strict transform of the exceptional curve of the first blowing-up and the image $\sigma\left(\widetilde{D}_{j}\right)$ is a singular point of the cubic curve $\sigma_{*} \widetilde{D}$.

Now since $\sigma_{*} \widetilde{D}_{j}=0, \widetilde{D}_{j}$ is the strict transform of $\sigma_{k}^{-1}\left(z_{k}\right)$ for some $k$ with $1 \leqq k \leqq 9$. However, $H_{i}$ and $\sigma_{i}^{-1}\left(z_{i}\right)$ intersect transversally at one point for $2 \leqq i \leqq 9$, since $H_{i}$ is smooth for $1 \leqq i \leqq 8$. Thus the strict transform of $\sigma_{i}^{-1}\left(z_{i}\right)$ on $Z$ has self intersection number -1 or -2 for $2 \leqq i \leqq 9$. One sees that $k=1$.

If $\sigma\left(\widetilde{D}_{j}\right)$ is a smooth point on $\sigma_{*} \widetilde{D}$, then $\sigma_{1}^{-1}\left(z_{1}\right)$ is not a component of $D_{1}$, and $\widetilde{D}_{j}$ is not a component of $\widetilde{D}$, which is absurd.

Proposition 6.2. Assume that $\widetilde{D}$ contains a component $\widetilde{D}_{j}$ with $\widetilde{D}_{j}^{2}=-3$ and $\widetilde{D}_{j} \cdot \widetilde{H}=1$. Then there is an admissible blowing-down $\sigma: Z \rightarrow \boldsymbol{P}^{2}$ to $\boldsymbol{P}^{2}$ such that $\sigma_{*} \widetilde{D}_{1}$ is a quadratic curve and such that the center $z_{1}$ of the first blowing-up does not lie on $\sigma_{*} \widetilde{D}_{1}$.

Proof. Case (1). Assume that there is an admissible blowing-down $\sigma: Z \rightarrow \boldsymbol{P}^{2}$ such that $\sigma_{*} \widetilde{D}_{1}$ is a cubic curve.

The curve $\sigma_{*} \widetilde{D}_{1}$ has a unique singular point $z$, since the reduced irreducible cubic curve $\sigma_{*} \widetilde{D}_{1}$ is the image of the rational curve $\widetilde{D}_{1}$. If $z=z_{1}$, then it contradicts the assumption $\widetilde{F} \cdot \widetilde{D}_{1}=2$. Thus $z \neq z_{1}$. If all of $z_{2}, \cdots, z_{9}$ are different from $z, \widetilde{D}_{1}$ is never smooth. Thus one element in $z_{2}, \cdots, z_{0}$ coincides with $z$. By exchanging the order of blowing-ups we can assume that $z=z_{2}$. Next if there is an element in $z_{3}, z_{4}, \cdots z_{9}$ which does not lie on the strict transform of $\sigma_{*} \widetilde{D}_{1}$, then we have $\widetilde{D}_{1}^{2}>-3$. Thus in particular $z_{3}$ lies on the strict transform of the cubic curve $\sigma_{*} \widetilde{D}_{1}$. The line passing through $z$ and $z_{1}$ does not intersect with the strict transform of $\sigma_{*} \widetilde{D}_{1}$ on $Z_{2}$. Thus this line does not contain $z_{3}$. Thus three points $z_{1}, z_{2}, z_{3}$ are not colinear. Now by considering the quadratic transformation with center $z_{1}, z_{2}, z_{3}$ we can reduce Case (1) to the next Case (2).

Case (2). Assume that the image $\sigma_{*} \widetilde{D}_{1}$ by an admissible blowing-down $\sigma: Z \rightarrow P^{2}$ is a quadratic curve.

If $z_{1} \in \sigma_{*} \widetilde{D}_{1}$, then we have $\widetilde{F} \cdot \widetilde{D}_{1}=1$, which contradicts the assumption. Thus $z_{1} \notin \sigma_{*} \widetilde{D}_{1}$.

Case (3). Assume that $\sigma_{*} \widetilde{D}_{1}$ by $\sigma: Z \rightarrow P^{2}$ is a line.

In this case the equality $\widetilde{F} \cdot \widetilde{D}_{1}=2$ never holds. Thus this case never takes place.

Case (4). Assume that $\sigma_{*} \widetilde{D}_{1}=0$ by $\sigma: Z \rightarrow \boldsymbol{P}^{2}$. 
If $\widetilde{D}_{1}$ is the strict transform of the exceptional curve of the first blowing-up, then we have $\widetilde{F} \cdot \widetilde{D}_{1}=1$, which contradicts the assumption. If $\widetilde{D}_{1}$ is the strict transform of the exceptional curve of the blowing-up on and after the second one, then $\widetilde{D}_{1}^{2} \geqq-2$, which is absurd. Thus case (4) never takes place.

Q.E.D.

Now for reader's understanding we give examples in the sequel.

EXAMPLE 6.3. An example of a reduced irreducible quartic surface with 2 triple points.

Consider a plane quartic curve $\bar{H} \subset \boldsymbol{P}^{2}$ whose singularity is a unique $A_{1}$-singular point at $P_{1} \in \bar{H}$. Let $L_{1}$ be a general line passing through $P_{1}$. Let $P_{2} \in L_{1} \cap \bar{H}$ be an intersection point with $P_{2} \neq P_{1}$. Choose a sufficiently general line $L_{2} \subset P^{2}$. Let $P_{3}, P_{4}, P_{5}, P_{6}$ be the intersection points of $L_{2}$ and $\bar{H}$. Furthermore let $L_{3}$ be a general line passing through $P_{3}$. We denote by $P_{3}, P_{7}, P_{8}, P_{9}$ the intersection points of $L_{3}$ and $\bar{H}$. Under this situation let $\sigma: Z \rightarrow P^{2}$ be the blowing-up of nine points $P_{1}, P_{2}, \cdots, P_{8}$. Consider the image $X$ by the morphism $\varphi=\varphi_{\widetilde{H}}: Z \rightarrow \boldsymbol{P}^{3}$ defined by the strict transform $\widetilde{H}$ of $\bar{H}$ by $\sigma$. The surface $X$ is quartic. We denote by $L_{1}^{\prime}, L_{2}^{\prime}$, and $L_{3}^{\prime}$ the strict transform of $L_{1}, L_{2}$ and $L_{3}$ by $\sigma$ respectively. Set $L_{4}^{\prime}=\sigma^{-1}\left(P_{3}\right)$. The image $\varphi\left(L_{1}^{\prime}\right)=\varphi\left(L_{4}^{\prime}\right)$ is a line and $\varphi\left(L_{1}^{\prime}\right)=\varphi\left(L_{4}^{\prime}\right)=$ Sing $X . \varphi\left(L_{2}^{\prime}\right)$ and $\varphi\left(L_{3}^{\prime}\right)$ are points and they are triple points lying on Sing $X$.

EXAMPLE 6.4. The Plücker surface.

This is the surface discussed in Klein [17]. It is rational.

Let $G$ be a smooth elliptic curve. We give it a group structure by choosing the origin $o \in G$. Let $p \in G$ be one of points of order 2. Set $E=\mathcal{O}_{G}(o-p) \oplus \mathcal{O}_{G}$ and $\bar{Z}=\boldsymbol{P}(E)$. Let $\pi: \bar{Z} \rightarrow G$ denote the projection and let $G_{0}$ and $G_{\infty}$ be the section of $\pi$ associated with the projections $E \rightarrow$ $\mathscr{O}_{G}(o-p)$ and $E \rightarrow \mathcal{O}_{G}$ respectively. It is not difficult to see that $Z=$ $\bar{Z}-G_{0}-G_{\infty}$ has a group structure such that $\pi \mid Z: Z \rightarrow G$ is a group homomorphism. Let $i: Z \rightarrow Z$ be the morphism associating an element with its inverse one. The morphism $i$ has 8 fixed points. Moreover we can extend $i$ to $\bar{Z}$ and the isomorphism $\bar{i}: \bar{Z} \rightarrow \bar{Z}$ is induced. Note that $\bar{i}\left(G_{0}\right)=G_{\infty}$ and $\bar{i}\left(G_{\infty}\right)=G_{0}$. The line bundle $L=\pi^{*} \mathcal{O}_{G}(o+p) \otimes \mathcal{O}_{\bar{Z}}\left(G_{0}+G_{\infty}\right)$ is invariant by $\bar{i}$. One sees that $L$ induces an morphism $f: \hat{X}=\bar{Z} / \bar{i} \rightarrow \boldsymbol{P}^{3}$. Here $\hat{X}=\bar{Z} / \bar{i}$ is the quotient variety. Setting $\hat{G}=\left(G_{0} \cup G_{\infty}\right) / \bar{i}$, the restriction $f \mid \hat{G}$ is a morphism of degree 2. However, the restriction $f \mid Z / i$ to $\hat{X}-\hat{G}=Z / i$ is an embedding. The image $X=f(\bar{Z} / \bar{i})$ has the one-dimensioal singular locus along the line $f(\widehat{G})$ and $X$ has $8 A_{1}$-singular point outside 
$f(\hat{G})$. This $X$ is the Plücker surface.

In [17] Klein showed that there is the duality between 8 isolated singular points on $X$ and 8 "singular tangent planes".

Remark. In [16] Klein discussed the Kummer surface $Y$. A quartic surface in $\boldsymbol{P}^{3}$ whose singularity is $16 A_{1}$-points is called a Kummer surface. It is known that any Kummer surface is the quotient of an Abelian surface by the involution induced by the group structure. When we fix an arbitrary line $l$ in $P^{8}$, we have 4 intersection points with $Y$ and 4 tangent planes to $Y$ containing $l$. If we divide 4 intersection points to 2 sets of 2 points, then it is known that a division of the above 4 planes to 2 sets of 2 planes is induced. Then he defined that 2 points in one of 2 sets are conjugate to 2 planes in one of 2 sets. He studied this notion "conjugate" closely in [16].

\section{References}

[1] A. Altman and S. Kleiman, Introduction to Grothendieck Duality Theory, Lecture Notes in Math., 146, Springer, Berlin, 1970.

[2] M. ARTIN, On isolated rational singularities of surfaces, Amer. J. Math., 88 (1966), 129-136.

[3] A. Beauville, Complex Algebraic Surfaces, Cambridge Univ. Press, Cambridge, 1983.

[4] J. W. BRUCE and C. T. C. WALL, On the classification of cubic surfaces, J. London Math. Soc., 19 (1979), 245-256.

[5] A. CleBsCH, Ueber die Abbildung algebraischer Flächen, insbesondere der vierten und fünften Ordnung, Math. Ann., 1 (1869), 253-316.

[6] M. Demazure, Surfaces de Del Pezzo, Séminaire sur les singularités des surfaces, Lecture Notes in Math., 777, Springer, Berlin, 1980, 21-69.

[7] A.H. DuRfese, Fifteen characterizations of rational double points and simple critical points, Enseign. Math. II, Ser., 25 (1979), 132-165.

[8] T. Fujita, On the structure of polarized varieties with 4 -genera zero, J. Fac. Sci. Univ. Tokyo, 22 (1975), 103-115.

[9] T. Fujita, On the structure of polarized manifolds with total deficiency one, I. J. Math. Soc. Japan, 32 (1980), 709-725.

[10] T. Fujita, On polarized manifolds of $\Delta$-genus two; part I, J. Math. Soc. Japan, 36 (1984), 709-730.

[11] F. Hidaka and K. Watanabe, Normal Gorenstein surfaces with ample anti-canonical divisor, Tokyo J. Math., 4 (1981), 319-330.

[12] C. M. Jessop, Quartic Surfaces with Singular Points, Cambridge Univ. Press, Cambridge, 1916.

[13] M. KATo, Riemann-Roch theorem for strongly pseudo-convex manifolds of dimension 2, Math. Ann. 222 (1976), 243-250.

[14] M. Kato aND I. NarukI, Depth of rational double points on quartic surfaces, Proc. Japan Acad. Ser. A, 58 (1982), 72-75.

[15] M. KATO and I. NARUKI, On rational double points on quartic surfaces, preprint RIMS390, Kyoto Univ., Kyoto, 1982.

[16] F. KLEIN, Uber Konfigurationen, welche der Kummerschen Fläche zugleich eingeschreiben 
und umgeschreiben sind, Math. Ann., 27 (1885), 106-142.

[17] F. KLEIN, Uber die Plückershe Komplexfläche, Math. Ann., 7 (1874), 208-211.

[18] E. Kummer, Ueber die Flächen vierten Grades auf welchen Schaaren von Kugelschnitte liegen, J. für reine und ang. Math., 64 (1865), 66-76.

[19] M. NötheR, Ueber die rationalen Flächen vierter Ordnung, Math. Ann., 33 (1889), 546-571.

[20] H. C. Pinkham, Simple elliptic singularities, Del Pezzo surfaces and Cremona transformations, Proc. Sympos. Pure Math., 30, A.M.S., Providence, 1977, 69-71.

[21] K. RoHN, Ueber die Flächen vierter Ordnung mit dreifachen Punkte, Math. Ann., 24 (1884), 55-151.

[22] K. SaIto, Einfach elliptische Singularitäten, Invent. Math., 23 (1974), 289-325.

[23] C. SEGRE, Etude des différentes surfaces du $4^{\circ}$ order à conique double ou cuspidale (générale ou décomposée) considérées quadratiques de l'espace à quatre dimensions, Math. Ann., 24 (1884), 313-444.

[24] J. SHAH, Degenerations of K3 surfaces of degree 4, Trans. Amer. Math. Soc., 263 (1981), 271-308.

[25] Swinnerton-Dyer, An enumeration of all varieties of degree 4, Amer. J. Math., 95 (1973), 403-418.

[26] T. TAKahashi, K. Watanabe and T. Higuchi, On the classification of quartic surfaces with a triple point, part I, part II, Sci. Rep. Yokohama Nat. Univ. Sect. I, 29 (1982), 47-70, 71-94.

[27] Y. UMEzU, On normal projective surfaces with trivial dualizing sheaf, Tokyo J. Math., 4 (1981), 343-354.

[28] Y. Umezu, Quartic surfaces of elliptic ruled type, Trans. Amer. Math. Soc., 283 (1984), $127-143$.

[29] Y. UMEZU and T. URABE, The appendix to [13].

[30] T. URABE, On quartic surfaces and sextic curves with singularities of type $\tilde{E}_{8}, T_{2,3,7}$, $E_{12}$, Publ. R. Inst. Math. Sci. Kyoto Univ., 20 (1984), 1185-1245.

[31] T. URABE, On quartic surfaces and sextic curves with certain singularities, Proc. Japan Acad. Ser. A, 59 (1983), 434-437.

Present Address:

Department of Mathematics

FACULTY OF ScIENCES

TOKyo Metropolitan University

Fukazawa, Setagaya-kU, Tokyo 158 